\title{
Lapurdum
}

Euskal ikerketen aldizkaria | Revue d'études basques |

Revista de estudios vascos | Basque studies review

$4 \mid 1999$

Numéro IV

\section{Itsassoco biayetaco othoicen araldea «La série de prières pour les voyages en mer » de Joannes Etcheberri de Ciboure (1627)}

\section{Aurélie Arcocha-Scarcia}

\section{(2) OpenEdition}

Journals

Édition électronique

URL : http://journals.openedition.org/lapurdum/1527

DOI : 10.4000/lapurdum.1527

ISSN : 1965-0655

Éditeur

IKER

Édition imprimée

Date de publication : 1 octobre 1999

Pagination : 9-44

ISBN : 2-84127-156-0

ISSN : $1273-3830$

Référence électronique

Aurélie Arcocha-Scarcia, «Itsassoco biayetaco othoicen araldea

"La série de prières pour les voyages en mer » de Joannes Etcheberri de Ciboure (1627) », Lapurdum

[En ligne], 4 | 1999, mis en ligne le 01 avril 2010, consulté le 29 janvier 2020. URL : http://

journals.openedition.org/lapurdum/1527 ; DOI : 10.4000/lapurdum. 1527 


\title{
ITSASSOCO BIAYETACO OTHOICEN ARALDEA "LA SÉRIE DE PRIÈRES POUR LES VOYAGES EN MER" DE JOANNES ETCHEBERRI DE CIBOURE (1627)
}

\section{Présentation et organisation du discours}

\author{
Escaldunen ozpea da lur guztira hedatcen, \\ Eta itsashuri ontçat munduac tu laudatcen \\ "La renommée des Basques s'étend à la terre entière, \\ Et le monde les honore en tant que bons mariniers."
}

Joannes ETCHEBERRI (1627/1669)

Joannes Etcheberri de Ciboure est un auteur dont on sait fort peu de choses, qu'il ait été frère de Piarres Detcheverry dit Dorre de Saint-Jean-de-Luz (Haristoy, Vinson, Moreau 1992, 269)' , n'est pas envisageable si l'on situe sa naissance à la fin du XVI ${ }^{e}$ siècle (Altuna 1981, 8). Par ailleurs, les Archives Municipales de Saint-Jean-de-Luz nous apprennent que "Pierre Detcheverry dit Dorré" est pilote et qu'il a 53 ans en janvier $1690^{2}$. Il serait né, par conséquent, en 1636 ou 1637, soit un ou deux avant la mort de Juannes Etcheberri (1638) et dix ans après la première impression du Manual Deuotionezcoa (1627) où figure le texte Itsassoco biayetaco othoitcen araldea, "Série de prières pour les voyages en mer."

Joannes Etcheberri de Ciboure aurait appartenu plutôt à la génération du grand-père de Piarres Detcheverry dit Dorre, Saubat Detcheverry dont l'épouse, Marie de Larralde, était décédée avant 1629. Le père de Piarres Detcheverry, Sansin Detcheverry de la maison Dorrea, s'était marié en premières noces le 5 février 1629, avec Marie Doyambéhère (Lassus, 1999) ${ }^{3}$. Nous ne connaissons pas la date de la mort de Piarres Detcheverry dit Dorre mais, en tout état de cause, il était encore vivant en 1690 et a dû mourir entre les dernières années du siècle et les toutes premières du suivant.

Joannes Etcheberri et Piarres Detcheverry dit Dorre ont cependant vécu tous deux dans des localités qui se jouxtent et tous deux sont liés, d'une façon ou d'une autre, à l'activité maritime ${ }^{4}$, ils pourraient donc fort bien avoir appartenu à la même famille.

Les divers paratextes en latin (le permis de publier accordé par M. D'Oiharard le 28 octobre 1626, les hommages à l'auteur rendus par P. Guillentena, I. de Heguy et Stephanus Hirigoity) nous apprennent qu'il est Doctor Theologo, ce qui 


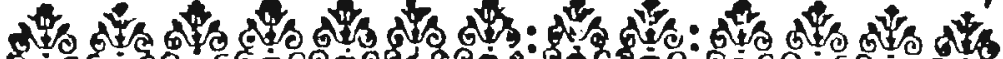

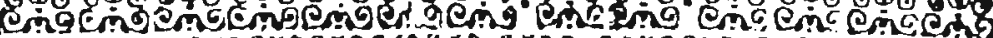

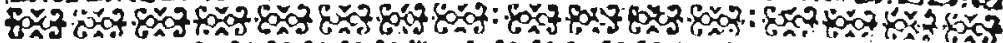

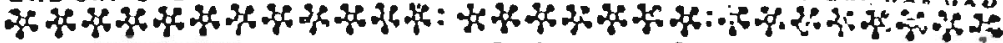

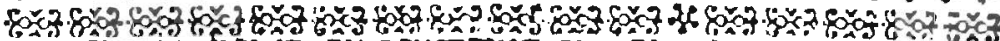

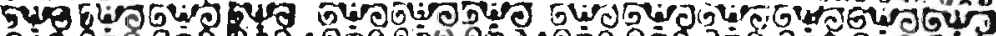

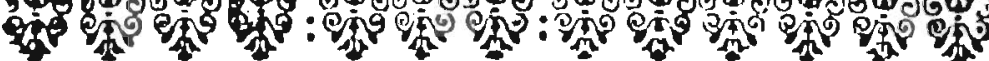

\section{T SAS SOC O \\ B I A Y ET A C O \\ O T HOI TCE N A R A L D E A}

\section{DEDICATIO NE PARTICVLARA ERRAN $D E N$ I $V$ N.}

MIGVELOIHARARTVICARIO General Official eta Calonge Theologal berari.

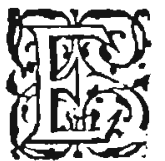

Scaldunen ozpea da lur guztira hedatcen, Era irfalturiontçat munduac tu laudarcen. Ecen hain behcracziu Oceanac ondarra, Nondic athera berunaz ezteçaren legarra. Eta eztu yrbazterric errelacac heçatcell. Norat bidea chuchenquin ezbaitute aurkhitcen. Norce cta Hegoaco hartac dire lekhuco. 
laisse entendre qu'après avoir étudié chez les jésuites à Pau où il a fait ses Humanitate, il a été à l'université comme deux autres auteurs fondamentaux du même siècle pour les lettres basques, Pedro Daguerre Azpilcueta "Axular", qui a fait ses études à l'université de Salamanque mais n'a pas atteint le grade de docteur en théologie (Altuna 1981, 8), et Arnaud Oihenart qui a obtenu le grade de licencié à la faculté de droit de Bordeaux le 7 septembre 1612. Reste à savoir quelle est l'université qu'il a fréquentée. Il faut tout de même dire que la faculté de théologie était, depuis le XIII siècle, la plus importante des trois facultés supérieures (les deux premières étant, par ordre croissant d'importance, celle de médecine et de droit) et que des études fort longues (et coûteuses) étaient nécessaires avant de pouvoir postuler le doctorat ou maîtrise en théologie (Imbach, Méléard 1986, 19).

\section{La critique d'Oihenart :}

Chacun sait que l'historien, collecteur de proverbes et poète à ses heures, Arnaud Oihenart n'appréciait pas les talents poétiques de Joannes Etcheberri qu'il jugeait ainsi :

"Il Est dommage que Cet Esprit aye mieux aymé s'apliquer à la poésie (pour laquelle Il nauoit point de naturel) qu'à la prose, En laquelle II auroit reussy Indubitablement ; car (outre ses ouurages Imprimes) ses Lettres familiaires, Escrites a aucuns de ses amys, son dictionnaire, Et ses conjugaisons (que Iay Ueus Escrits de sa main) rendent temoignage de sa suffisence en Cette langue." (Oihenart 1665/1997, 207)

Vingt-huit ans ans après la publication du Manual, à un moment où le mouvement amorcé par la Pléiade à la fin du XVI siècle, se fige jusqu'à devenir une véritable "législation" élaborée selon les canons d'une "esthétique de l'autorité" (Aquien 1993, 10), Oihenart conteste, on l'a vu, à Joannes Etcheberri la virtuosité formelle du poète classique. Il est intéressant de noter que son interprétation ("Ce qui faict Iuger..."), est fidèle à la théorie contenue dans l'Art Poétique où hors le respect de certaines règles formelles bien définies par lui (élisions, synérèse, raccourcissement des mots, attention portée aux rimes longues et brèves, rimes qui doivent toujours dépasser une syllabe et demi etc.), le poète basque ne doit point espérer de salut (Oihenart 1665/1997, 201).

Toutes ces "licences poétiques", intolérables pour Oihenart, se retrouvent souvent dans le Manual de Joannes Etcheberri, de même que l'omniprésence de ce vers de quinze syllabes qui l'irrite particulièrement. Mais, outre le Manual qui est critiqué de long en large, exemples à l'appui, c'est, en conclusion, la totalité de l'œuvre qui sera très sévèrement jugée par Oihenart :

"La façon de ses Noels, de son Egunoroscoa, Et de son Eliçara erabiltzecoa nest pas meilleure. Il y faict rimer doloraturen contre Iaquinen galdetcen contre çuten, galduric contre norbaitic, garbi contre humillqui, Et Il se donne, en plusieurs lieux, dautres Licences Exorbitantes, Et compose aussy quelque fois sa rime dUn mesme mot reiteré a la fin des deux Uers, Et, a fin datraper La mesure de ses Uers, il redouble souuant larticle Entre ladjectif et le substantif, comme Lors quil dict, Enea galdea pour /Ene galdea/, pensamenduetaric gaxtoetaric pour pensamendu gaxtoetaric. Au reste Il ne faict que rarement dElision ny de Synaerese, Et les Uers y sont de quinse syllabes, aussy bien que ceux du manuel ; 
nonobstant quils soient distribues en quattre Uersets; car de ces quattre ny en ayant que deux qui riment, Les autres deux doyuent estre pris pour des hemistiques, et non pour des vers Entiers." (Oihenart 1665/1997, 207)

Sans entrer dans la polémique, tel n'étant pas le propos de ces lignes, signalons toutefois que, outre le succès qu'il eut en son temps, les chercheurs contemporains ont mis en lumière la qualité expressive de Joannes Etcheberri. Pierre Lafitte, par exemple, qui a un avis opposé à celui d'Oihenart sur son aptitude "naturelle" à la poésie :

"Etcheberri fut fameux en son temps, et à juste titre : mots, images, rimes coulaient de source chez lui, et la magnificence du verbe n'empêchait pas un profond réalisme." (Lafitte 1941, 37).

Luis Michelena suit, tout d'abord, l'argumentation d'Oihenart (il aurait mieux fait d'écrire en prose), mais remarque cependant que "sus dotes de expositor claro y preciso son extraordinarios" (Michelena 1961/1988,76); vingt ans plus tard, il souligne que Joannes Etcheberri a écrit en vers la majeure partie du Manual devotionezcoa en toute connaissance de cause et en vue d'un objectif précis (Mitxelena 1988, 121). Avis sur lequel est revenu récemment Bernard Oyharçabal.

Par ailleurs, Patxi Altuna soupçonne Oihenart de faire un procès d'intention injuste à Joannes Etcheberri, auteur célébré en son temps, et qu'il critique plus que tout autre dans son Art Poétique (Altuna 1981, 16). Jean-Baptiste Orpustan, enfin, trouve à l'auteur une "dextérité évidente et une facture élaborée dans l'emploi des vers courts et la disposition des rimes" et ce, malgré "le reproche de rimes trop pauvres" que lui fait Oihenart (Orpustan 1996, 43).

\section{Des prières destinées aux mariniers :}

La deuxième partie du Manual debotionezcoa où figure Itsassoco biayetaco araldea ou "La série de prières pour les voyages en mer" n'a pas bénéficié d'une édition critique comme ce fut le cas avec la première partie (Altuna 1981) et n'est pas directement disponible puisque la seule réédition facsimilée existante depuis 1667, celle de 1978, est épuisée depuis longtemps. Une telle situation est évidemment regrettable, elle s'explique peut-être par un a priori négatif, cette seconde partie étant considérée de moindre qualité que la première :

"Bi zati nagusi ditu, aski desberdin elkarren artean, ez neurriz, baina bai balioz, ene ustez, lehenak bigarrenari alde handia daramaiolarik poesiaz eta goi argiz."

"Il a deux parties [le Manual...], assez dissemblables entre elles; non par la dimension, mais, à mon avis, par la valeur, la première étant bien supérieure à la seconde en ce qui concerne la qualité poétique et l'inspiration." (Altuna 1981, 10).

Pour Pierre Lafitte, toutefois, le texte Itsassoco biayetaco othoicen araldea qui figure dans la deuxième partie offre un grand intérêt ethnographique et historique :

"[...] trente pages de son Manuel sont consacrées à la vie du marin : il y a des prières pour les phases les plus diverses de la pêche : découverte de la baleine, harponnage, résistance de la bête, mort de la proie, noyade d'un compagnon, et le reste; bref une foule de détails qu'on chercherait en vain ailleurs sur les pêcheurs basques au XVII siècle." (Lafitte 1941, 37) 


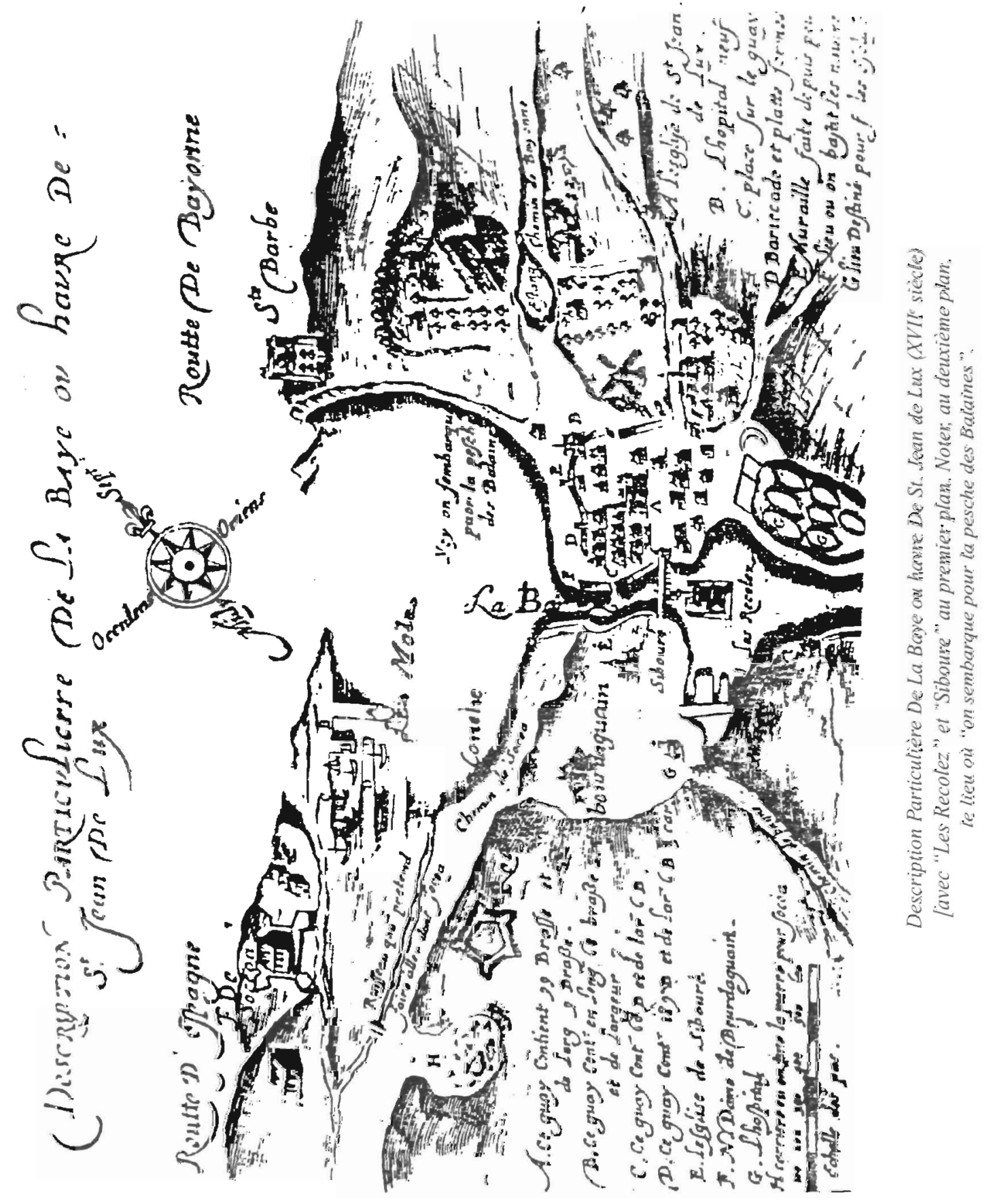


Luis Michelena suit en cela Pierre Lafitte :

"Para el lector actual sea los más sugestivo la grandeza de ciertos pasajes dedicados a los Novísimos y la minuciosa información que facilita sobre la vida de su tiempo y en particular de la de los hombres de mar. Etcheberri, como su pueblo natal, miraba más al Atlántico que a la tierra firme que quedaba a sus espaldas." (Michelena 1961/1988, 76)

Oihenart, dans son Art Poétique, avait aussi mentionné le même texte pour y glisser un jugement négatif implicitement lié au lectorat non savant formé par les mariniers, destinataires privilégiés de Itsassoco biayetaco othoicen araldea:

"Ie lui ay ouy dire au temps qu'il composoit ses Vers qu'il trauaillait principalement pour les mariniers Lesquels les Chantoient sur la mer; Ce qui faict Iuger qu'il Escriuoit plus tost par Un motif de Charitté que par aucune ambition, ou Vainegloire, Et quil auoit le zèle de profiter à son prochain que doit avoir Un Veritable Ecclesiastique." (Oihenart 1665/1997, 207)

Oublions, pour l'heure, le jugement discutable d'Oihenart à propos des motif's qui pousseraient Joannes Etcheberri à écrire, pour retenir simplement le début de la phrase où l'on apprend que ces prières ont été chantées sur des navires au moins entre 1627 (date de publication du Manual) et 1665 (date de rédaction de la lettre d'Oihenart).

La question de l'oralité se pose puisque de telles prières étaient destinées à être entendues et écoutées.

\section{Des prières à écouter, à dire, à chanter et des livres à lire en basque sur les navires :}

A l'évidence, Pierre Lafitte n'accordait pas le statut de "cantiques" aux prières de Joannes Etcheberri :

"Joannes Etcheberri de Ciboure, docteur en théologie, fait paraître trois livres de piété en vers: Manuel (sic) devotionezcoa (1627), Noelac (1631) et Eliçarat erauilceco (1636). Cette idée étonne un peu, quand il ne s'agit pas de cantiques. L'homme en prière doit-il hausser le ton, arrondir sa phrase et rythmer avec élégance les cris de sa pitoyable misère ? Jadis on se plaçait à un autre point de vue : on voulait soigner son style par décence, par respect pour Dieu et on ne pensait pas que ce fût un luxe de "prier sur de la beauté". (Lafitte 1941, 37)

Villasante s'étonne aussi du choix opéré par Joannes Etcheberri :

"Los tres [libros] sont religiosos y los tres están compuestos en verso. Hoy nos puede parecer un tanto extraño el que para orar y pedir a Dios se recurra al verso. Etcheberri se excusa de ello alegando la gran afición que tiene el vasco al verso, y por aquelle época también en otras literaturas se encuentran ejemplos parecidos." (Villasante 1961, 70)

La technique adoptée par Joannes Etcheberri, qui s'était pourtant expliqué, serait essentiellement due à l'analphabétisme du lectorat (Mitxelena 1981, 1988, 121) mais Oyharçabal n'en est pas convaincu : 
"Mitxelena (1981) fonde sur ces indications l'hypothèse selon laquelle, contrairement à Axular, Etcheberri avait écrit ses vers pour un public qui ne lisait pas, af in de lui en faciliter l'apprentissage. [...] je reste pour ma part dubitatif, malgré l'autorité des auteurs mentionnés, l'un à titre d'analyste [Mitxelena], le second en tant que rapporteur des propos de l'auteur [Oihenart], sur l'exactitude de cette interprétation. La versification d'Etcheberri, même dans le second livre du Manuel qui est considéré comme littérairement plus modeste $[\ldots]$, n'est certainement pas celle que l'on attendait de textes destinés à être mémorisés facilement : les inversions, avec antéposition des syntagmes adnominaux ou adjectivaux au vers précédent, de manière à obtenir la rime, abondent, comme si le basque était du latin. [...] Certes les vers d'Etcheberri ont eu un grand succès populaire, et certains de ses cantiques (notamment de Noelak) se chantent encore dans les églises du Pays Basque. De même, la nature pédagogique de l'ouvrage est incontestable, et son titre même l'indique. Pour autant il me semble difficile de ne pas prêter à l'auteur une ambition allant audelà d'une mise en couplets simplement destinée à faciliter la mémorisation chez un public analphabète. [...]

Faut-il les penser comme des textes devant être appris par cœur et récités ou chantés dans ces circonstances ainsi que le dit l'auteur? Ne sommes-nous pas en présence plutôt d'un positionnement littéraire dans le cadre d'un ouvrage dépassant en fait sa nature fonctionnelle?" (Oyharçabal 1999)

Ceci dit, y a-t-il vraiment contradiction entre la finalité orale dévolue aux textes concernés et la "présence d'un positionnement littéraire" ?

Dans le premier quart du XVII ${ }^{e}$ siècle, la dispositio du texte n'est-elle pas encore souvent indissociablement liée à l'elocutio et à l'actio ? Autrement dit, le processus ne fonctionne-t-il pas dans les deux sens ? Ce qui doit être prononcé, récité, chanté (ces deux derniers procédés sont parfois fort proches), ne peut-il pas être écrit selon les règles de la rhétorique, et, inversement, la mémorisation n'est-elle pas facilitée par une dispositio complexe du texte écrit mettant en mouvement plusieurs coordonnées (position des chapitres, sémiotique des titres, répétitions et parallélismes etc.), dispositio à laquelle s'ajoute une elocutio facilitée par une structure mélodique perdue à jamais pour nous en ce qui concerne les textes étudiés et une actio inhérente au rituel de la prière dont nous trouvons quelques indications éparses dans les paratextes ou les textes introductifs tant chez Joannes Etcheberri que chez Materre comme nous le verrons ultérieurement.

Les textes de Joannes Etcheberri ne pouvaient-ils pas ainsi être appris par cœur malgré certaines particularités syntaxiques semblant précisément faire obstacle à la mémorisation? La question se pose.

Nous avons perdu irrémédiablement toute la dimension charnelle de la voix. On peut écouter un témoignage peut-être de cet ancien monde dans le $O i$ Eguberri Gaua, écrit il y a plus de 300 ans par Joannes Etcheberri et toujours chanté, parfois de mémoire, dans la plupart des églises du Labourd, de BasseNavarre et de Soule le soir de Noël. 
Se pose aussi la question du degré d'alphabétisation du lectorat dont l'analphabétisme serait bien moindre qu'on ne le pense généralement entre la fin du $\mathrm{XVI}^{\mathrm{e}}$ et le début du XVII ${ }^{e}$ siècle. (Oyharçabal 1996, 38,39)

Plusieurs pilotes labourdins devaient bien savoir lire en basque en 1677 pour que Piarres Detcheverry, dit Dorre ait éprouvé le besoin de publier la version basque, des Voyages Avantureux (1579 ?) du capitaine Hoyarsabal de Ciboure ${ }^{6}$ ? Il avait d'ailleurs actualisé l'ouvrage en fonction de la découverte de nouveaux lieux dans l'immense île que les Labourdins nommaient ternua (les "terres neufues"). Cette destination lointaine et dangereuse était annuellement prise par des navires partis de Saint-Jean-de-Luz ou de Bayonne, navires dont l'armement se faisait souvent à Bordeaux, La Rochelle, voire plus au nord et d'où ils entreprenaient la grande et périlleuse traversée de l'Atlantique Nord qui pouvait être très rapide (autour de deux semaines) ou très longue (plus d'un mois), selon les caractéristiques du navire et surtout les conditions atmosphériques.

Un passage de la Dotrina Christiana de Esteve Materre ${ }^{7}$ publiée en 1617 (seconde édition en 1623, troisième édition, avec changement de titre et adjonctions, en 1693), mérite attention à cause des indications sur le processus rituel de la prière à bord des navires basques ${ }^{8}$ qu'il fournit. Le passage en question est dirigé aux marins qui doivent réciter des prières en mer, MARINELEC NOla eguin behar diluzten bere othoilçac itsasoan dabilçan denboran, "COMMENT LES MARINS doivent faire leurs prières pendant le temps où ils sont en mer", il s'agit des plus anciennes prières connues en basque (et latin) consacrées aux marins ${ }^{9}$. Elles précèdent de dix années le Manual de Joannes Etcheberri ${ }^{10}$.

Les précisions données par Esteve Materre peuvent ainsi nous aider à comprendre comment se déroulait l'actio et la dictio des prières de Joannes Etcheberri à bord des navires basques durant le premier quart du siècle.

Après avoir précisé son dessein en préambule :

Ceren bait-taquit iende añhits dela Euscal-herrian itsasoan dabilanic, behar derauet hei ere eracutsi nola behar derautçate $[n] "$ here oracinoac, eta othoitçac Iaincoari eguin... "Car je sais que nombreux sont ceux qui en Pays basque vont sur mer, je m'en vais à eux aussi leur apprendre comment ils doivent dire leurs oraisons et leurs prières à Dieu...". (Materre 1617/1693, 219)

Esteve Materre indique quel est le rituel à suivre pour la prière du matin :

Goicean demborac hala emaiten duenean çuecitsasoan çabiltçaten marineloc biltçaitezteelcar gana iar çaitezte belhaurico, eta ceuen gogoac Iaincoa gana ailtchaturic eta ceiñaturic, erran eçaçue : Pater noster : Aue Maria : Eta Credo Latinez edo Euscaraz.

"Au matin, si le temps le permet, vous marins qui allez sur les mers, rapprochez-vous les uns des autres, agenouillez-vous, et, après avoir élevé vos âmes vers Dieu et vous être signés, dites : pater noster : Ave maria : et Credo en latin et en. basque."

Puis ajoute ceci :

"Eta hauquen ondoan erran beça çuetaric batec appurbat gora eta haguitz orai hemen ibenico dudan othoitza : Eta bitartean eduqui beçate bertcec ere har- 
DOTRINA CHRISTIANA.

BIGARREN IMprefsionean debocinozco ot oitz eta qracino atcuez betret:cric.

Aita Esteve Materre San Francifcoren Ordenaco Fraideac hirur partetan eguina.

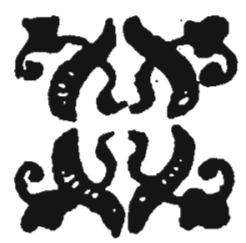

\section{BORDELEN,}

IAceVES MIILANGES, Erregueren Imprimaçaillearenean. 2.623 . 
tan gogoa, ençun beçate, edo norc bere liburuetan ichilic iracur beçate, ceren estimatcen dutez içanen bat-ere iracurtcen daquien murinelic liburu haurc içanen eztuenic."

"Et que par la suite l'un d'entre vous lise d'une voix assez haute la prière que je vais disposer à présent ici : et que les autres, pendant ce temps, concentrent aussi là-dessus leur esprit, qu'ils écoutent, que chacun lise dans son livre en silence, car j'estime qu'il n'y aura aucun marin, parmi ceux qui savent lire, qui ne possédera le présent livre." (Materre 1617/1693, 220)

Le texte montre bien que la lecture des prières se fait encore habituellement "à voix assez haute", appurbat gora, par un seul lecteur, (la lecture à voix basse étant récente).

Fait remarquable, l'auteur mentionne aussi comment doivent se comporter les autres, les "écoutants", dont certains sont d'ailleurs "écoutants-lecteurs" ! Il leur est en effet expressément ordonné de "Iire en silence", ixilic iracur beçate (aujourd'hui, au contraire, la lecture à voix haute serait à préciser puisque c'est: en silence que tout le monde lit).

Toutes ces indications confirment que plusieurs marins savent lire en basque au début du XVII ${ }^{c}$ siècle et que plusieurs, si l'on en croit le texte cité, ont manifestement un livre entre les mains. Ils sont donc alphabétisés ${ }^{12}$

Mais arrêtons-nous un instant sur les objectifs de Esteve Materre. Il dit avoir écrit la Dotrina Cristiana pour deux raisons. Une raison pastorale, tout d'abord:

Eta nola lanac cimendutic behar baitu assi, eta gure salbemenduco obrarem cimendua baita Dotrina Christiana, halatan nic ere, handic hasten naicela, hurtu dut gogo liburutto hunen eguiteco, eta iendeartera atheratceco [...], "Et comme le travail doit commencer par le fondement, et que le fondement de notre ouvre salvatrice est la Doctrine Chrétienne, c'est ainsi que moi aussi, commençant de la même manière, $\mathrm{j}$ 'ai eu l'intention de faire ce petit livre et de le divulguer parmi les gens $[\ldots]$.

Il indique aussi que le livre peut servir à enseigner la doctrine chrétienne berce eracusleric eztenean, "quand il n'y a pas d'autre maître". Une idée que Luther et Calvin avaient comprise et mise en pratique dès les débuts du siècle précédent...

La deuxième raison invoquée par Materre se situe en dehors du cadre religieux et concerne l'alphabétisation en basque.

Eta guero ikus dadin halaber nola behar den Euscara esquiribatu eta iracur$t u$, "Et de même, pour qu'on voit ensuite comment il faut écrire et lire le basque."

Julien Vinson s'appuie sur cette phrase pour dire que "Le P. Materre ne paraît pas avoir vu de livres basques antérieurs au sien, pas même Liçarrague." (Vinson $1898,54)$

Oyharçabal n'est pas aussi catégorique (Baina horrek Leizarragaren itzulpenak ez zituela batere begistatu erakusten ote $d u$ ? "Mais cela montre-t-il qu'il n'avait jamais lu les traductions de Liçarrague ?")

La conclusion de Vinson semble effectivement quelque peu hâtive car à aucun moment Esteve Materre ne fait référence aux livres en basque lus ou non lus, ni 
à l'existence (ou non) de prédécesseurs. Hormis les paroles ci-dessus, il ne fait que deux autres allusions au fait d'écrire en basque :

1/ EVSCALDUNEI/Miretsico duçue aguian nic (Euscal-herico ez naicelaric) Euscaraz esquiriatceco ausartciaren hartcea., "AUX BASQUES. Vous vous étonnerez peut être que, n'étant pas du Pays basque, j'aie l'audace écrire en basque." (Materre 1617/1693, A3)

2/ Eta etçait iduri asco de la Cadiratic predicatuz probetchu eguitea baina are vstedut esquiribuz ere bear de la enseiatu eta trabaillatu, "Et il ne me semble pas que tirer bénéfice du sermon fait en chaire soit suffisant, je pense, au contraire, qu'il faut aussi essayer et travailler en écrivant." (Materre 1617/1693, A3)

Ces deux passages, ainsi que ceux cités précédemment, peuvent tout aussí bien être interprétés en sens contraire, c'est à dire comme un témoignage de plus sur la circulation de livres écrits en basque au Labourd.

L'exhortation à lire, et la promotion de son propre ouvrage qu'Esteve Materre fait en s'adressant aux gens de mer, dévoilent la présence de livres chez les marins basques du Labourd au XVII ${ }^{e}$ siècle. Marins qui passent, sans transition, et avec l'aisance que confère une longue habitude, du latin au basque (Latinez edo Euscaraz, "en latin ou en basque", dit Materre), le français n'étant jamais évoqué... La mention faite, vers la fin du siècle, par le jésuite Bernard Gasteluçar de Ciboure (1619-1701) qui avait mis "en vers des hymnes latins pour que les marins basques puissent les chanter" ${ }^{13}$ dans son livre Eguia Catolicac saluamendu eternalaren eguiteco necessario direnac (1686), fournit à ce sujet un témoignage exceptionnel :

Eta hek prinzipalki konsideraturik, deliberatu dut liburu hunen egitera ; erranen baitute batzuek, bizi zenean orhoitu zen bere herriaz, eta ez zitzaion ahantzi hiltzerakoan, bere señalleak emanik joan baita. Ezen nola plazer harizen baitute itsasoan latiñez Elizako himnoen kantatzeaz, atsegin gehiago izanen dutela himno beren ezkaraz errateaz, aire berean ezkaraz ematen tut ; eta gaiñerako bersuen airea komunzki gustiek badakite. (Gasteluçar, Eguia Catolicac)

"Et c'est en ayant principalement ces derniers [les marins basques] à l'esprit que j'ai décidé d'écrire ce livre. Ainsi certains diront : lorsqu'il vécut, il se souvint de son pays, et il ne l'oublia pas non plus à l'heure de mourir, car il est parti en laissant son empreinte. En effet, comme les marins prennent plaisir à chanter en latin les hymnes de l'église lorsqu'il sont en mer, je les traduis en basque sur le même air, pensant qu'ils auront encore plus de plaisir à les dire en basque ${ }^{14}$.

Le livre de dévotion, livre imprimé, maniable et pratique, peut rendre de grands service au marin entouré par d'immenses périls. Périls physiques indissociables du péril spirituel dans la mentalité de l'époque. L'âme non instruite à la parole divine est en effet en grand danger de se perdre face aux périls de la mer. L'apprentissage des prières est donc fondamental pour le marin.

Dans une charte de 1741 concernant le "reglement particulier entre les capitaines, pescheurs de baleines" qui comprend 11 clauses, on peut observer que la récitation des prières vient au premier rang! Elle peut nous aider à savoir comment se déroulait le processus un siècle auparavant. En voici le début : 


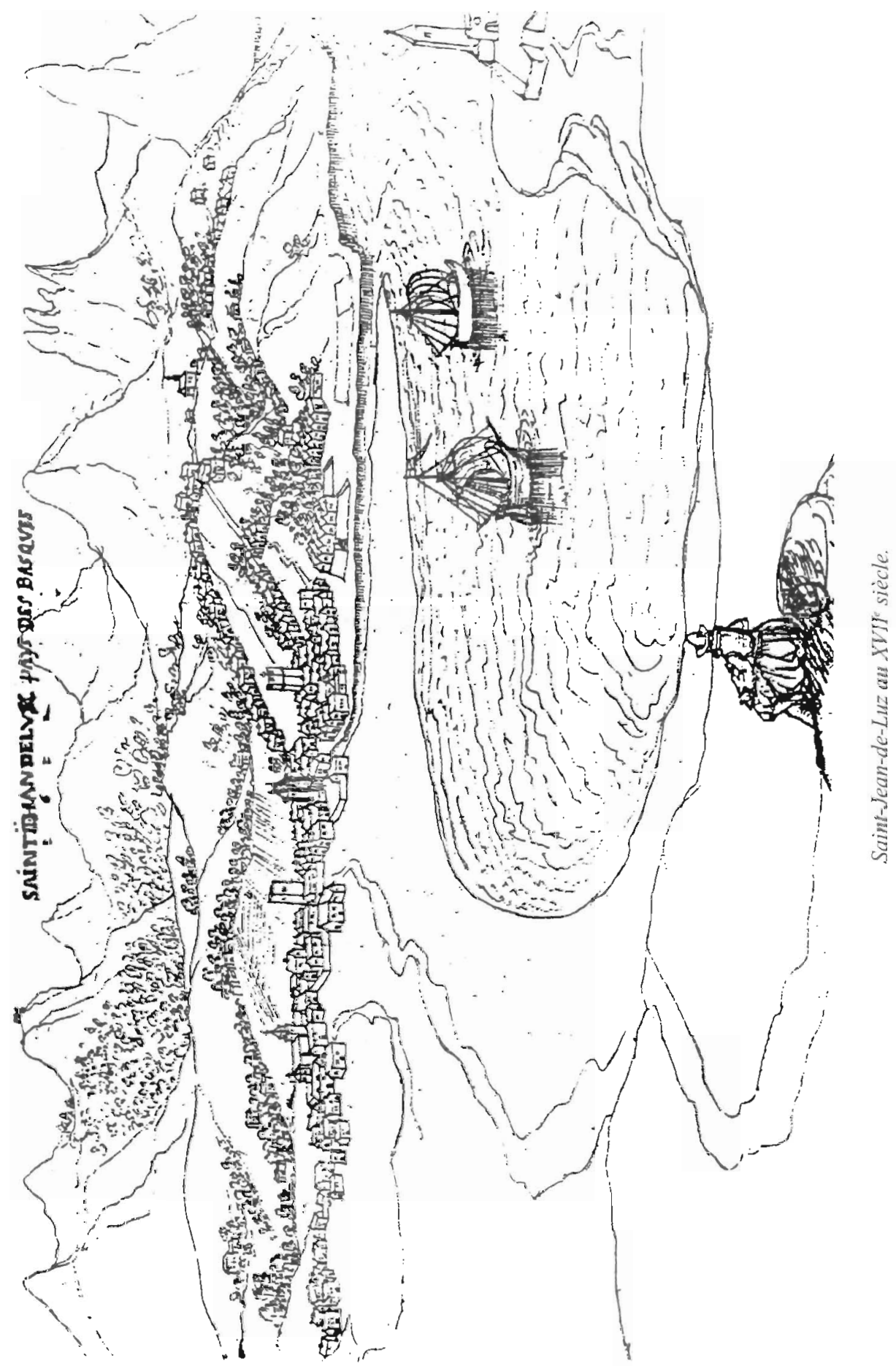


"Nous, Officiers et Matelots, nous sommes loués au Capitaine N. N... commandant le navire pour aller cette année à la pêche à la baleine, pour le pays auquel chacun de nous est convenu, promettant d'obéir sur toutes choses au dit Capitaine, tant en mer qu'à terre, et à celui qui lui succédera s'il vient à mourir ;

$1^{\circ}$ ) Nous promettons de nous rendre le matin et soir, aux prières et de les écouter avec dévotion et modestie, à peine de payer amende que le Capitaine ordonnera;" (Jaupart 1981)

Esteve Materre mentionne expressément la nécessité d'avoir des marins d'autant plus "éveillés", iratçarragoac, et "instruits" ikassiagoac, à la parole divine "qu'ils encourent plus de périls en mer", periletan ago baitabiltça itsasoan ${ }^{15}$...

Mais restituons le passage dans son entier:

... eta egun oroz haren Majestateari bere burua gomendatu. Eta eguia erraitera premia handiaco gauçada haur, Ceren combatenaz, eta halacoac periletan ago baitabilça itsasoan, han batenaz laincoari gomendatceco iratçarragoac, eta ikassiagoac behar dute egon Hequentçat bada eçarten [dit] ut hemen othoitz eguiteco moldeac ceiñetaz probetcha ahal ditezque egun oroz, hambat goicean nola arratsean."

“... et se recommander tous les jours à sa Majesté [celle de Dieu]. Et, à dire vrai, il s'agit là d'une chose très urgente, car ils [les marins] doivent être d'autant plus éveillés et instruits à se recommander à Dieu qu'ils encourent de grands périls en mer. Pour eux donc je place ici les manières de faire les prières dont ils pourront tirer profit tous les jours, le matin comme le soir." (Materre, 1617/1693, 219)

C'est par la prière en effet que l'âme du marin en danger se dirige vers le port métaphorique du salut (Axular).

Le livre lu ou écouté est précieusement conservé. C'est qu'il contient des renseignements pratiques indispensables, ceux fournis par les "routier des mariniers" et les cartes adjointes qui les complètent, sont indispensables au pilote qui doit aborder les côtes lointaines.

Mais les livres de dévotion sont tout aussi nécessaires pour les marins de l'époque. C'est ainsi que Joannes Etcheberri peut écrire, Ecen ardiastecotçat beharrezco premia/ Sinhetçac othoitza dela gustiz moian handia, "Pour obtenir la satisfaction du besoin pressant/ Crois, que la prière est un moyen très puissant") $)^{16}$.

\section{Des prières à mémoriser :}

Esteve Materre et Bernard Gasteluçar montrent in situ et presque in vivo, comment pouvait se dérouler le processus de la lecture, de là récitation et du chant en groupe à bord des navires. Même lorsque le texte n'était pas chanté, cela supposait de la part du lecteur, une certaine scansion, un certain rythme où la ponctuation jouait bien entendu un rôle essentiel (de Libera, 842). 
Joannes Etcheberri s'exprime plus volontiers sur la lecture solitaire. Le contenu de "I'Avis au lecteur" (Iracvrtçailleari abisva) de la première partie du Manual est très parlant ne serait-ce que par les conseils didactiques donnés et la description du cérémonial solitaire de la prière :

"Ilhabete oro behin iracurrac guztia

Iesus Christoc munduari declaratu eguia.

Noicic behingoac ere, heure eguin bidera,

Atçartureneau manuen ereduz bizitcera.

Baiñan othoitz eguiteco, apartean lekhua,

Iduquiçac çarţ̧o eta soiñutic vrrundua.

Paretean hedaturic, Imagina ederqui,

Eta vr benedicatua bazterrean chahuqui."

"Lis en entier une fois par mois

la vérité déclarée au monde par Jésus Christ.

Une lecture occasionnelle également te poussera à ton devoir,

A vivre selon les commandements.

Mais tiens le lieu de tes prières à part,

Éloigné du tapage et de la musique,

L'image sainte joliment déployée sur le mur,

Et l'eau bénite placée proprement sur le côté." 17

La rhétorique de la mémorisation est mentionnée de façon encore plus explicite dans le deuxième avertissement figurant en tête du deuxième livre :

Eta beraz ikhaz eçac lañcoaren othoizten

Eta oren guztietan behar denaz ert[c]hatcen

hartaracotz hemen diat antcea declaratu,

Eta haren eredura exempluac moldatu.

Asqui dena ene penec liquetec irabaci,

Baldin asti orduetan nahi baituc ikhassi.

Bañan errac astiroqui othoitcetan lehenic,

Othoiztea cein baitago hurren plaman etciñic.

Ecen othoitcen han diat moldea arrimatu,

eta Iañcoari mintço natçaiola, moldatu.

Hunec ernaturen daroc bihotça othoiztera,

Pentsatcean, nolaco den, othoizteco manera.

"Et, donc apprends à prier Dieu,

et à toute heure à l'implorer de ce qui est nécessaire.

Pour cela j'ai ici exposé une méthode,

Et suivant ce modèle établi des exemples.

Mes peines auront été assez récompensées,

Si durant tes loisirs tu veux les apprendre.

Mais dis d'abord lentement parmi les prières

Celle qui est couchée dans le page suivante.

Car là j'ai placé la manière de prier,

Que j'ai composée comme une adresse personnelle à Dieu." 18

Dans "L'Avis au marin lecteur" (Itsasturi iracvrtzailleari abisua), c'est encore la mémorisation qui est visée, rappelons qu'il s'agit de la quatrième partie de l'éloquence avec l'invention, l'élocution, la disposition et l'action qui comprend la prononciation...

Si, pour tout un chacun, cette mémorisation peut se faire durant les loisirs, asti denboretan, comme nous venons de le voir, elle peut donc implicitement s'effectuer aussi à terre, avant l'embarquement, pour celui "qui se destine à être marin". 


\begin{abstract}
Ceren vrtan ohi diren gaitz handiac guerthatu. han dabillanac choill maiz dicahoan Iongoicoa, Illkhi eta badu ere ahantzten ondicoa.

Hemen tiat bada othoitz batçuc hangotz formatu,

Mariñel behar duenac gogoan detçan hartu.

"Car souvent de grands malheurs sont arrivés sur l'eau.

Qui fréquente seul ces lieux-là invoque souvent Dieu,

Même si après s'en être sorti il oublie l'infortune.

C'est pourquoi j'ai conçu quelques prières pour ces contrées-là,

Que celui qui se destine à être marin les prenne en mémoire."
\end{abstract}

\title{
VUne typologie du discours lyrique?
}

Cet apprentissage qui peut finalement se faire seul, collectivement, à terre ou sur mer, permet l'acquisition d'une mémoire efficace qui se manifeste par une récitation sans failles, adaptée à toute les circonstances, comme en témoignent ces vers dédiés à Migvel Oiharart qui introduisent Itsassoco biayetaco othoitcen araldea :

Hemen bada formatutut othoitz baţuc ureco,
Behar aldi orduetan dewotqui errateco.
"J'ai donc élaboré quelques prière pour [à dire sur] l'eau,
Afin que l'heure du besoin venue on les dise dévotement."

Si l'on est en droit de parler du "positionnement littéraire" de Joannes Etcheberri (Oyharçabal 1999), il semble, répétons-le, qu'un tel positionnement ne puisse être séparé d'une oralité partie prenante de l'elocutio et de l'actio.

Mon point de vue s'appuie, bien entendu, sur la caractéristique de l'auteur, homme rompu à l'art oratoire, et sur les caractéristiques d'un texte "à dire", "à réciter", soit, mais hautement articulé du point de vue rhétorique. Il n'est que de relever diverses récurrences sur l'ordre ou l'ordonnancement au fil des divers paratextes pour s'en convaincre.

La mémorisation était liée aussi au chant puisque, si l'on en croit Oihenart, les marins chantaient les prières de Joannes Etcheberri.

Pourquoi donc avoir finalement choisi d'écrire des prières sous forme prosodique ? Parce que l'analphabétisme de son public l'y a contraint pour des raisons mnémotechniques?

Nous pouvons en tout cas remarquer que le premier des trois avertissements au lecteur en tête du Manual n'évoque à aucun moment l'analphabétisme mais bien l'amour de la copla qui existe chez les Basques, à quoi s'ajoute probablement son amour de la poésie grecque (Lehenago nonbait urrun Greçiaco partetan, "Jadis quelque part au loin dans les contrées de Grèce"...), fruit d'une éducation humaniste.

"Gvristinoa, eman diat escarazco versutan, Catholico manuala neure asti ordutan Ikhussirik nola bainaiz iatatcen escalduna ${ }^{19}$ Gure natioa dela copla maite duena. Hartaracotz iaquiara diat versus eçarri. Lasierrago ikhas eta maizago aipha garri. Lehenago nonbait vrrun Greçiaco partetan Herrico legueac eman ohi ituen cantetan 
Nola cantac maiz baitire munducoen ahoan,

Hala kantez orhoituric, maiz çitusten gogoan,

Hequin bada costuma onaz orhoituric lerroca

Manuala eman diat, den beçala coplaca.

Bañan nihorc eztemala beharria hitçera.

Hambat nola hitz azpian ehortçia denera."

$[\ldots]$

"Chrétien, j'ai écrit en vers basques,

Le manuel catholique durant mon temps de loisir,

Ayant observé, étant basque de naissance,

Que notre nation aime les couplets. ${ }^{20}$

Pour cette raison délibérément je l'ai mis en vers

Afin qu'il soit plus vite appris, et plus souvent mentionné.

Jadis quelque part au loin dans les contrées de Grèce

Les lois du pays étaient mises en couplets :

Comme les chants viennent souvent aux lèvres des gens,

Ainsi, en se souvenant de ceux-ci, ils avaient les lois fréquemment à

l'esprit, ${ }^{2}$

Me rappelant donc de leur bonne coutume, ligne à ligne,

J'ai mis en couplets le Manuel tel qu'il est.

Mais que nul ne prête l'oreille aux mots

Autant qu'il le fait à ce qui est enfoui sous eux." 22

La lyre d'Orphée n'est effectivement pas loin...

Bien que l'on puisse aujourd'hui légitimement mettre en question les catégorisations conventionnelles des genres littéraires (Todoror), il convient ici de se poser la question, ne serait-ce que pour clarifier la perspective adoptée dans le présent travail : s'agit-il de prières, de poésie lyrique?

Mais faut-il choisir entre ce qui fait partie đu "littéraire" et ce qui appartient au domaine du "non littéraire" ? Ne vaut-il pas mieux parler ici d'une "typologie du discours" (Todorov 1987,25$)^{23}$ où poésie lyrique et prière seraient réunies ? ${ }^{24}$.

Il est temps de rendre justice à ce petit livre de 31 pages très particulier qui figure dans la deuxième partie du Manual. Le peu d'attention dont il a été l'objet s'explique difficilement. En effet, hormis les trois prières se rapportant aux chasseurs de baleines qui sont souvent citées plus pour leur intérêt historique que littéraire, d'ailleurs, ces textes de Joannes Etcheberri qui pourtant ont une force lyrique et une qualité esthétique incontestables, restent injustement dans l'ombre.

A cela plusieurs raisons, nous l'avons dit : absence de réédition, d'édition critique, a priori négatif... Mais aussi, peut-être, une vision restrictive de la littérature, très éloignée, justement, des perspectives ouvertes adoptées par Tzvetan Todorov ou Roland Barthes par exemple.

Le routier des mariniers Biaya Venturosac (1677) de Piarres Detcheverry dit Dorre, à la fonction exclusivement pratique, les prières pour les marins que nous pouvons trouver dans Dotrina Christiana (1617) d'Esteve Materre, "la dévotion des marins" ${ }^{25}$ contenue dans Debocino Escvarra (1635) d'Haramboure (Vinson $1898,542,543$ ) ou la traduction en basque d'hymnes latins faite en 1686 par Bernard Gasteluçar dans Eguia catolicac ("Les vérités catholiques) témoignent d'une présence particulièrement dynamique du livre en basque chez les gens de mer tout au long du XVII siècle. 
Parmi tous les textes cités le texte Itsassoco biayetaco othoicen araldea contenu dans le Manual de Joannes Etcheberri est particulièrement original.

Cet article se veut une première approche, une première "lecture" de "La série de prières pour les voyages en mer". Prières, mais aussi "textes" présentés selon un "code", dans le sens ou la Bible est un great code (Fry 1984) et dans le sens où Roland Barthes analysant Les Exercices Spirituels d'Ignace de Loyola écrit :

"Tout "spirituels" qu'ils soient, les Exercices d'Ignace sont fondés en écriture. Il n'est pas nécessaire d'être jésuite, ni catholique, ni chrétien, ni croyant, ni humaniste, pour s'y intéresser." (Barthes 1971/1980, 46)

Les lignes suivantes seront consacrées à l'architecture textuelle élaborée par Joannes Etcheberri dans Itsassoco biayetaco othoicen araldea ou "La série de prières pour les voyages en mer". Il en sera donné une vision synthétique basée uniquement sur les paratextes du texte.

\section{L'organisation du discours dans Itsassoco biayetaco othoitcen araldea :}

\section{VI.1 - Avertissement}

Les 93 textes (dont 89 prières) de ce petit livre, inclus dans le grand Manual, qui a d'autres inclusions ou subdivisions très clairement mentionnées par Joannes Etcheberri ${ }^{26}$, se présentent selon une structure fermée tripartite avec une ouverture à deux volets dont le premier est constitué par la dédicace faite à Migvel Oiharart:

Dedicatione Particvlara erran den IAVN. MigVel oiharart Vicario General Official eta Calonge Theologal berari/"DÉDICACE AU DIT MIGVEL OIHARART VICAIRE Général Officiel et Chanoine Théologien."

Le deuxième volet étant constitué par ITSASTVRI IRACVRTÇAILILEARI ABISVA. ou "AVIS AU MARIN LECTEUR".

A ces deux paratextes peuvent s'ajouter "L'Avis", Abisua, placé au cinquième chapitre (Buru $V$ ) et le texte Itsassoco otoitcen çarratcea, "Fermeture [du cycle] des prières pour la mer" qui, malgré l'absence de caractères typographiques particuliers, est bien distinct des prières du corpus. Ce dernier texte est en réalité une conclusion qui "ferme" de cette manière un discours écrit selon les lois de la rhétorique. Il répond ainsi au deux paratextes du début et en particulier à Itsastvri Iracvrtçailleari Abisua ou "Avis au Marin Lecteur" qui est l'introduction la plus directe au corpus puisque, comme pour le dernier texte, le destinataire est uniquement le marin.

Afin de faire apparaître la "disposition" du texte entier, j'ai adjoint, de A à J, des lettres entre crochets [...] au têtes de chapitres et deux sortes de numérotation:

1. Une numérotation entre crochets [1], [6] etc., qui se rapporte à la construction de chaque chapitre en particulier et qui peut comporter des subdivisions dans des cas particuliers comme, par exemple, quand les cinq prières dévolues au Pilote sont énumérées. Les subdivisions apparaîtront en lettres alphabétiques minuscules et entre crochets, [a], [b] etc. 
2. Une numérotation entre parenthèses qui n'apparaîtra que dans le tableau II afin de ne pas alourdir la lecture. Cette numérotation prend en compte le nombre des prières (89) et ne se rapporte jamais aux paratextes déjà mentionnés, "Dédicace", les deux "Avis" et le texte terminal, qui font monter à 93 le nombre total de tex.tes.

A cette structure rhétorique de base, s'ajoutent trois (mais nous pourrions les réduire à deux) figures essentielles qui servent de "matériaux" de construction et de consolidation : l'antithèse, la gradation (ou climax) et son corollaire l'anticli$\max$.

L'antithèse a permis à l'auteur, souvent en les opposant deux à deux, de regrouper les prières, ce qui induit aussi des parallélismes et des répétitions très importants à l'heure de mémoriser.

Les antithèses seront indiquées dans le Tableau I avec les symboles A et non-A.

A ces antithèses s'ajoutent deux mouvements inverses d'un même principe dynamique : la gradation et l'anticlimax.

J'ai désigné les deux figures par deux symboles mathématiques. La gradation par $<$, soit, ce qui précède est "plus petit" que ce qui suit ; l'anticlimax par $>$, soit, ce qui précède est "plus grand" que ce qui suit.

Afin que le contenu sémantique des paratextes puisse être immédiatement lié à la construction du discours, les symboles utilisés, $\mathrm{A} /$ non- $\mathrm{A} /\langle/\rangle$ ne seront mentionnés que dans le Tableau I. L'utilité du Tableau II étant surtout de donner la synthèse de l'ordonnancement utilisé par Joannes Etcheberri avec numérotation des prières.

\section{VI.2 - Tableau I}

[A] BVRV I. Itsassoco gende guztiaren othoitçac [Viaya ona eguiteco]. "CHAPITRE I. "Les prières de tous les gens de mer [pour faire un bon voyage]."

$\begin{array}{cc} & {[1] \text { Viaya ona eguiteco. }} \\ & \text { "Pour faire un bon voyage" } \\ < & < \\ \text { A } \quad[2] \text { Viaya ona eguin dutenean. } \\ \text { "Lorsqu'ils ont fait bon voyage." } \\ <\quad \\ \text { non-A } \quad[3] \text { Viava gaichtoa eguitean. } \\ \text { "Quand on fait un mauvais voyage." }\end{array}$

[B] BVRV II. Particularen othoitçac [eta lehenic Burgessarenac]. "CHAPITRE II. "Les prières des particuliers [... et d'abord celles du Bourgeois].

[1] [eta lehenic Burgessarenac]

["et d'abord celles du Bourgeois" ${ }^{27}$ ] 


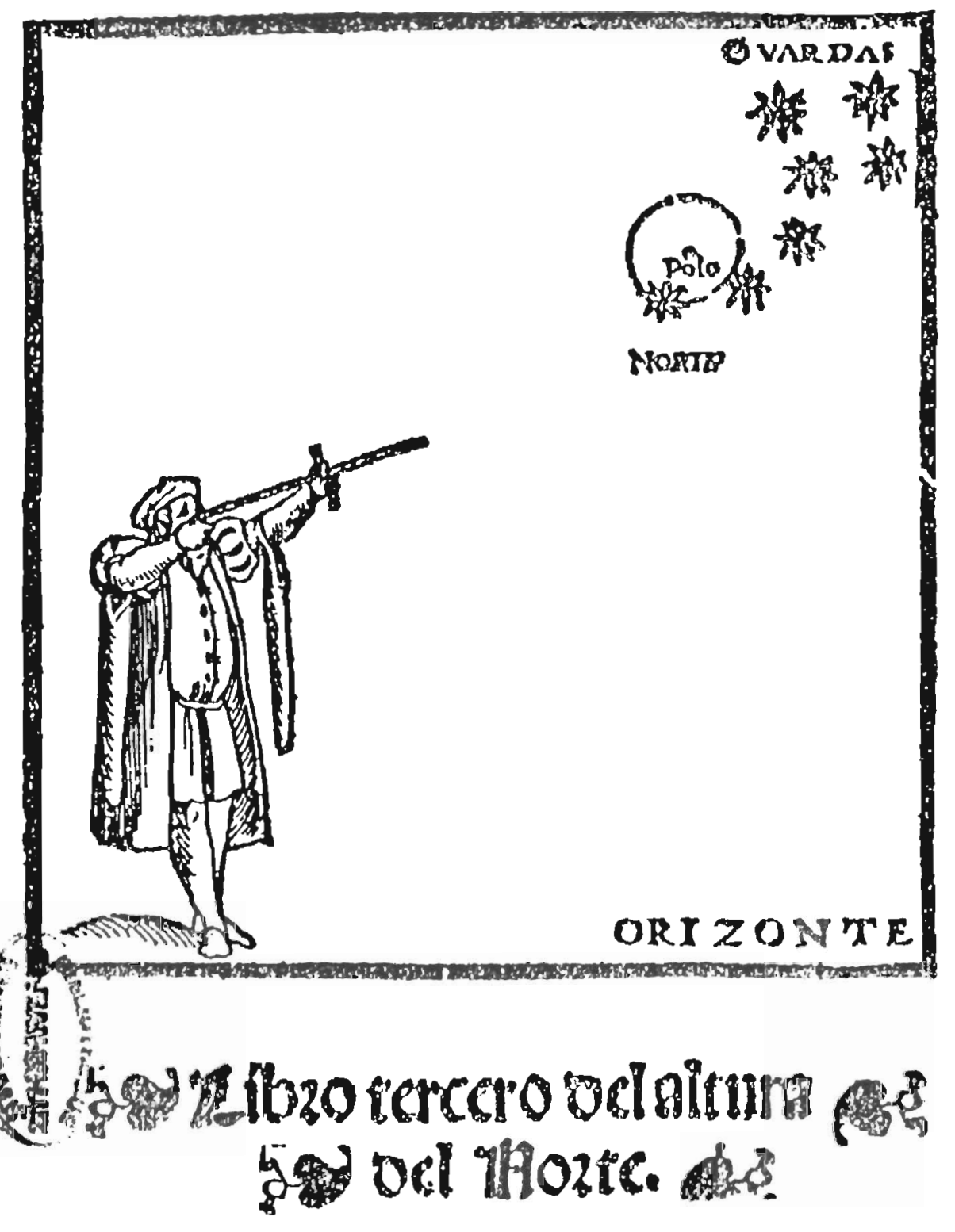

"Prendre hauteur" (XVI siècle) 
[2] Aguintarien othoitça.

"La prière des Officiers"

[3] Maistruaren othoitça.

"La prière du Maître." 29

[4] Pillotuaren othoitçac.

"Les prières du Pilote." 30

[a] Bercea

"Lautre"

[b] Arruta ematean.

"Quand on donne la route à suivre."

[c] Bideco contua hartcean.

"Quand on donne les coordonnées de la route." 31

[d] Haltura hartcean.

"Quand on prend hauteur." 32

[e] Carta miratcean.

"Quand on examine la carte."

[5] Lemacoac 3. bethiere.

"Celui qui tient le gouvernail" toujours [doit dire cette prière]."

A [a] Leman ${ }^{35}$ sartcean.

"Quand on entre dans la timonerie.

non-A [b] Lematic illkhitcean

Quand on sort de la timonerie."

[6] Lagunen othoitça.

"La prière des compitgnons."

\title{
[C] BVRV III. Vntciaren gatic guztiec eguiteco othoitçac.
}

"CHAPITRE III. Les prières que l'on doit faire à cause du navire."

\author{
A [1] Guilla ematean \\ "Quand on met la quille." \\ non-A [2] Vntcia çaharturic haustean. \\ "Quand le navire se brise par vieillesse." \\ A [3] Vntcia saluoric heldudenan. \\ "Quand le navire rentre sauf." \\ non-A [4] Vntçia galtcen bada. \\ "Si le navire se perd." \\ A [5] Vntçia buthatcean. \\ "Quand on lance le navire." \\ nom-A [6] Vntcia çaharturic encallatcean. \\ "Quand le navire s'échoue par vieillesse."
}




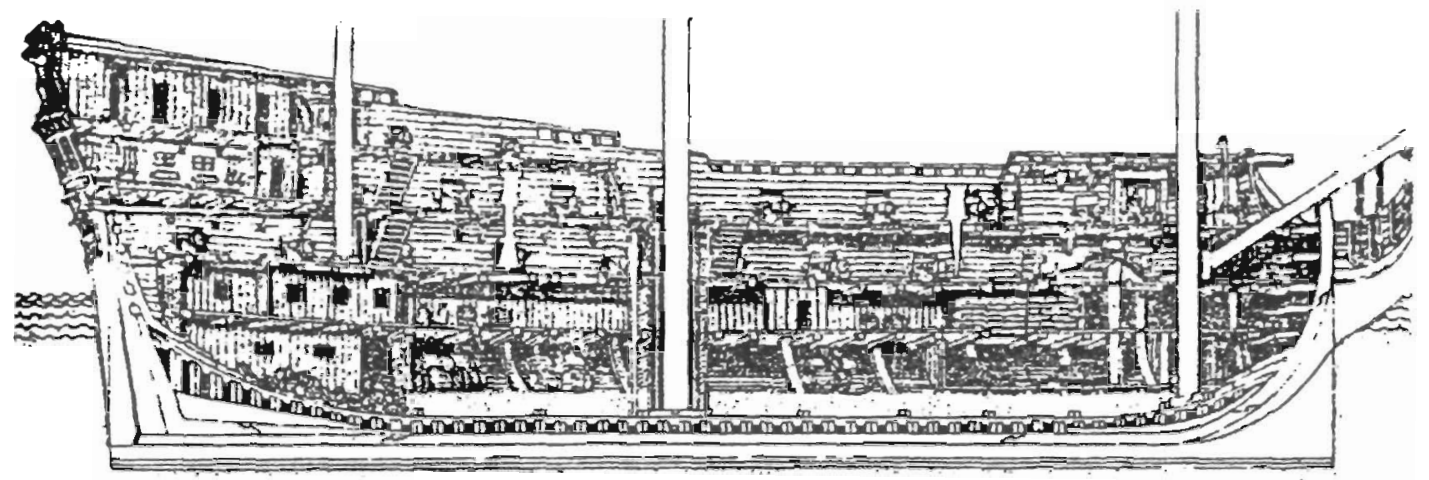

1. Quitle

2 Erion.

3. Etrave.

4. Figure.

5. Tatorn

6. Enmbat.

7. Contrequalie de

l'ment

Contraturave.

Contrequilite os Tarrize

10. Contrtambot.

11. Mrail?

12. Meitre cousple

13. Couple de lot de

\&
14. Couple de lof de larrize.

15. 15. Liree de hourdy.

16. 16. Berres diurcase.

17. Couroninement.

18. Volte

19. 19. Corlingre.

20. 20. Porquen

21. 21. Guirlader.

22. Fourches de maswene.

23. Rechenge du maitre canonnier.

24. Armoirea aux gargoessoch.
25. Le grand mit et les porspes dana l'urchipornpe.

26. Amoire ars garprousect de Tovant.

7. Planches a lis dis tribution aux rivrea.

28. Charabre du chirupgien.

29. Ecosutille pour le rechunge dus a non.

30. 30. Ecoutithe aur poudra.

31. Ecoutille our vivrea.
3232 Grande Econtille.

33. 33. Eocutille aux ablen.

34. Ecoutille de la fouse axu bona.

35. Ginnd cep de dric.

36. Mus de misaine.

37. Barredburavecaid

38. Mu dertimea

39. 39. Carand cabestan.

40. Petit abearen

41. Counin de beau. pre.
42. Eaubien.

43. 43. Panseaus crillebotia.

44. 44. Promoners par bo thelen.

45. Chambre due on mandhere.

46. Colta.

17. 47. Chembrea exiciern.

48. Cobene des pin

49. Meouelle du $y$ verneat.

50. Four.

51. 51. Riteliers mancarres.

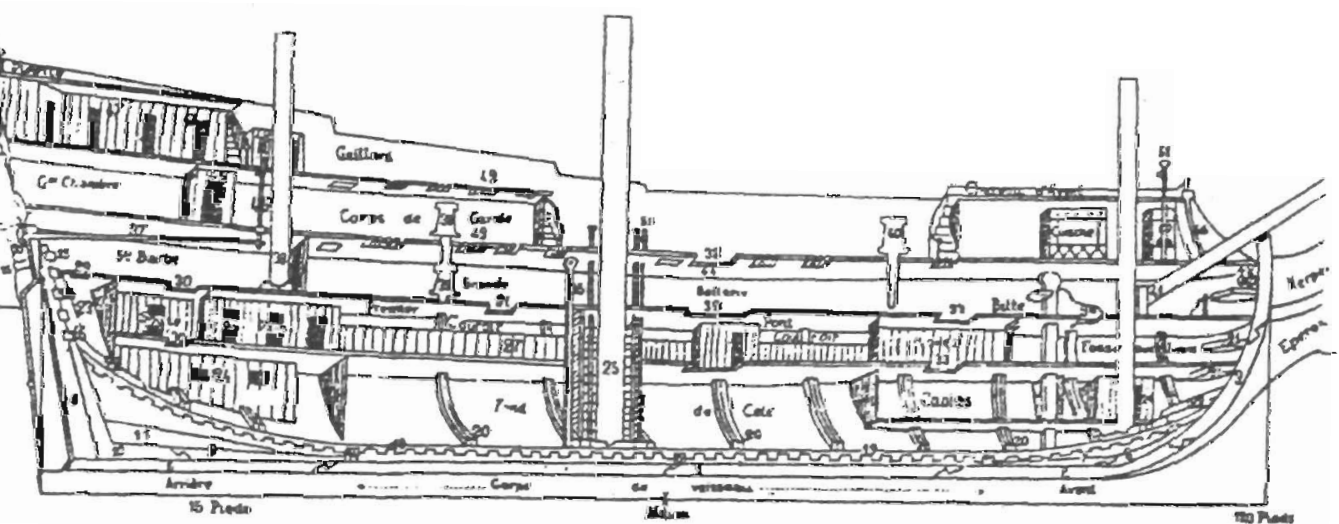




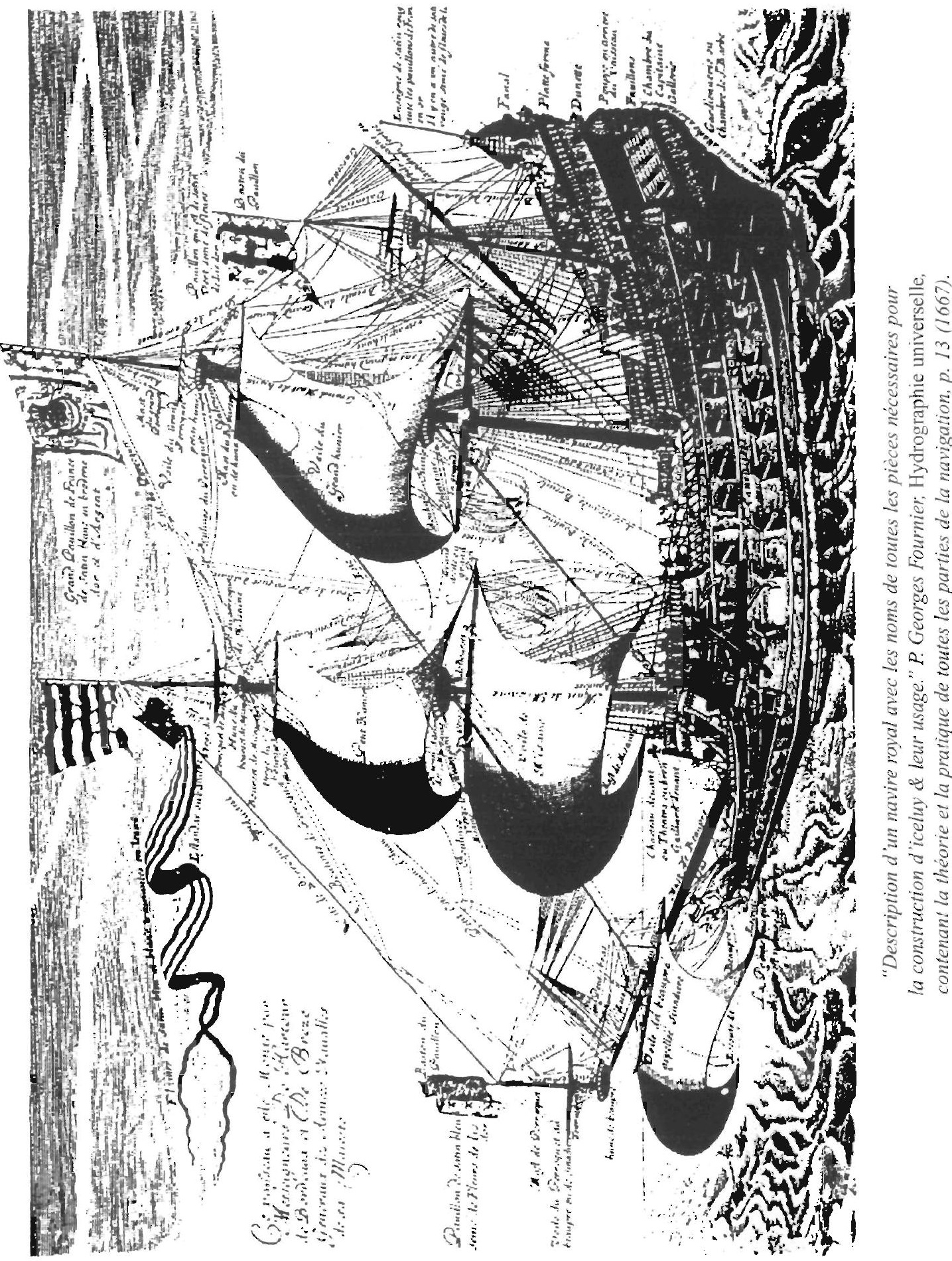




\section{[D] BVRV IV. Viaya bereco othoitçac. \\ "CHAPITRE IV. Les prières du voyage en soi."}

A [1] Embargatcean.

"Quand on embarque."

non-A [2] Desembargatcean.

"Quand on débarque.".

A [3] Aingurac altchatcean.

"Quand on lève l'ancre."

non-A [4] Aingura egotztean.

"Quand on jette l'ancre."

A [5] Belac hedatcean.

"Quand on déploie les voiles."

non-A [6] Belac hartcean.

"Quand on ramasse les voiles."

A [7] Partiada higuitcean.

"Quand on amorce le départ ;"

non-A [8] Vntcia bihurturic ${ }^{36}$ guelditcean

"Quand le navire qui est de retour s'arrête."

A 9] Herrico portutic illkhicean.

"Quand on sort du port du pays."

non-A [10] Portu berera iţ̧ultcean.

"Quand on entre au même port."

A [11] Lehorra ${ }^{37}$ estaltcean.

"Quand disparaît la terre ferme."

non-A [12] Berce lehorra aguertcean.

"Quand apparaît l'autre terre ferme."

A [ 13] Itsassoan edocein orduz.

"Sur la mer à n'importe quelle heure."

non-A [14] Berce untciarequin batcean.

"Quand on s'unit à l'autre navire."

A [15] Bidean cembait ${ }^{38}$ portutarat ezqueretcean.

"Quand en chemin il y déviation vers un port quelconque."

non-A [16] Portu hartaric illkhitcean.

"Quand on sort du dit port."

A [17] Portu noraco illkhi baitcen, han sartcean.

"Quand on entre au port d'où on était parti."

non-A [18] Portu hartaric bihurtcean.

"Quand on revient du dit port." 

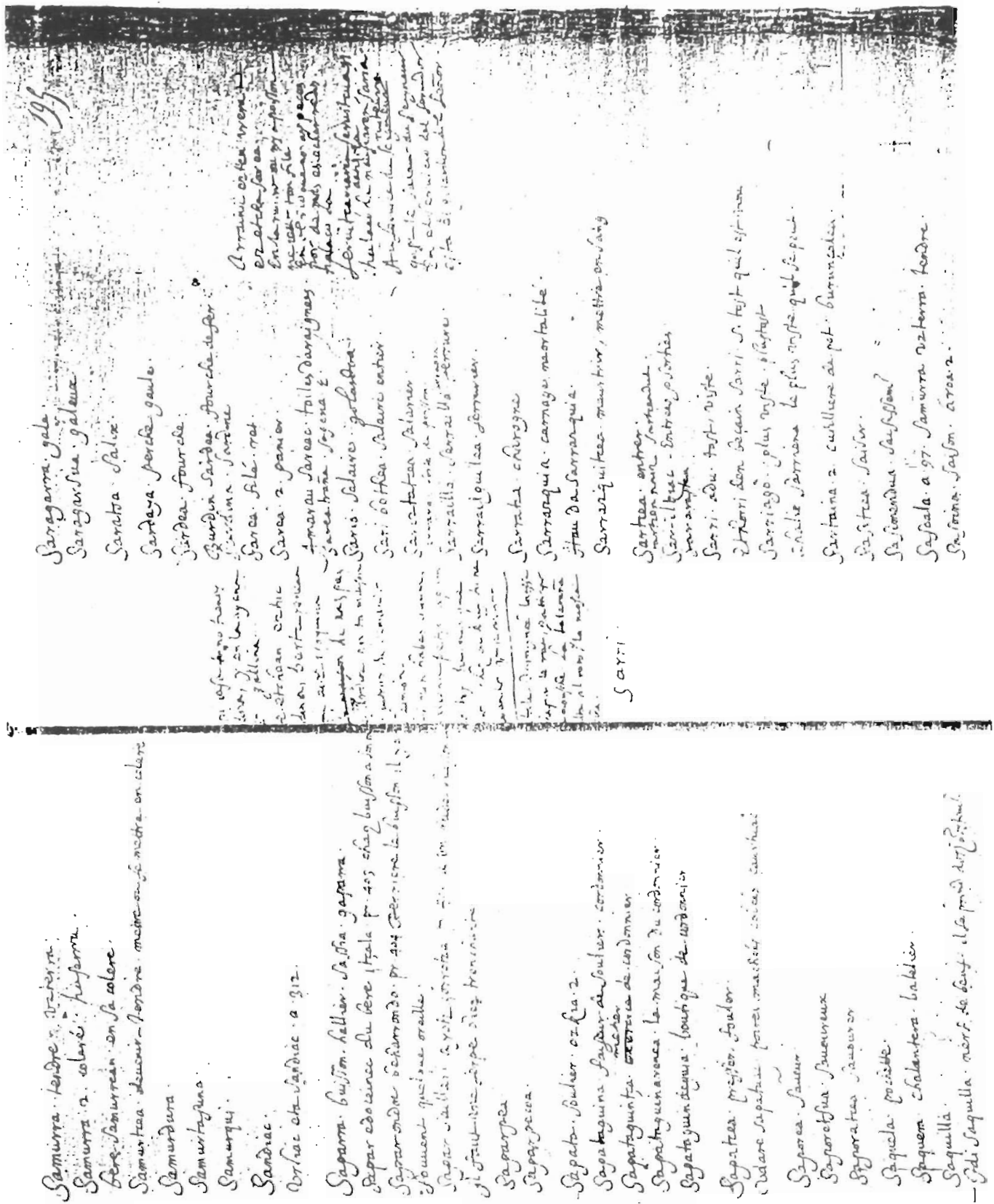
[E] BVRV V. Guardiaco othoitcez.

"CHAPITRE V. Des prières de la garde."

$$
\begin{aligned}
& \text { ABISVA } \\
& \text { "AVIS" } \\
& <
\end{aligned}
$$

[F] BVRVVI. Edocein ordutaco othoitçac [egunaz].

"CHAPITRE VI. Les prières pour toute heure [du jour] ${ }^{39}$ ""

$$
\begin{array}{cc}
\text { A } & \text { [1] [egunaz]. } \\
& \text { ["du jour"] } \\
\text { non-A } & \text { [2] Gauaz. } \\
& \text { ["De la nuit."] }
\end{array}
$$

[G] BVRV VII. Premiazco othoitçac [calman].

"CHAPITRE VII. Les prières en cas d'urgence [par temps calme]."

$$
\begin{aligned}
& \text { [1] [calman] } \\
& \text { ["par temps calme"] }
\end{aligned}
$$


[2] Continuatçen badu.

"Si ça continue."

[3] Haice coniracoa denean

"Quand le vent est contraire."

[4] Vria deneco.

"Pour quand il y a un grain."

[5] Goibel deneco.

"Pour quand il fait sombre."

[6] Tormenta denean.

"Par gros temps."

[7] Irauten badu.

"Si ça dure."

[8] Promessa Iaincoari

"Promesse à Dieu"

$>$

[9] Vntçiaren Patroñari.

"Au Patron du navire."

$>$

[10] Itsassoco berce Patroñei.

"Aux autres Patrons ${ }^{40}$ de la mer."

[11] Promessaren complitçeco.

"Pour accomplir la promesse."

[12] Vreziondoratçeco perillean.

"Quand il y a danger de s'abîmer au fond de l'eau."

[13] Khassatçen duenean.

"Quand il chasse."

[14] Vuntciac iotcen duenean edo encaillatcen denean.

"Quand le navire frappe [le fond] ou qu'il

s'échoue."

[15] Vntcico deusac egotztean.

"Quand on jette les choses du navire."

$<$

[16] Ecin portua hartuz penatcean.

"Quand [le navire] peine sans réussir à atteindre le port."

[17] Etsituric costaratcean.

"Quand, désespérés, on est emportés vers la côte." 
[18] Cur puchcan lehorrera venturatcean. "Quand sur le morceau de bois on s'aventure vers la terre ferme."

[19] Biciaz etsitceraco menean.

"Quand on est sur le point de perdre tout espoir de vivre."

[20] Biciaz etsitu eta.

"Une fois que tout espoir de vivre a été perdu."

A [21] Vntcitic nihor erori denean barrenecoec. "Quand quelqu'un est tombé à la mer, ceux du navire [disent cette prière]."

non-A [22] Vntcia bera galtceco perillean dabillanean gendeac salburic promessa.

"Promesse quand les gens sont saufs alors que le navire lui-même est. en danger de se perdre."

[24] Promessaren complitcecoa "[Prière] concernant l'accomplissement de la promesse."

\title{
[H] BVRV VIII. Arrantçale guztientçat othoitçac, [arrain atcemateco]. "CHAPITRE VIII. Les prières pour tous les pêcheurs, [afin qu'ils trouvent du poisson]."
}

\author{
[1] [arrain atcemateco] \\ ["afin qu'ils trouvent du poisson"] \\ A $\quad$ 2] Atcematen badute.
"S'ils en trouvent." \\ non-A [3] Atcematen ezbadute. \\ "S'ils n'en trouvent pas."
}

[I] BVRV IX. Arrantçale particularen othoitçac.

"CHAPITRE IX. Les prières des pêcheurs particuliers."

A [1] Arrain ttipi çaleentçat othoitça.

"Prière pour ceux qui s'adonnent à la pêche aux petits poissons."

non-A [2] Balea çaleenţ̧at.

"[Prières] pour ceux qui s'adonnent à la chasse à la baleine."

[a] Balea colpatu eta.

"Après avoir blessé la baleine."

[b] Balea hill ondoco esquerrac.

"Remerciements après la mort de la baleine." 
[J] BVRV X. Itsassoco viayetan hilltcen direnentçat othoitçac.

"CHAPITRE $X$. Les prières pour ceux qui meurent au cours des voyages maritimes."

\author{
A [1] Edocein uiayetan hilltcen denarenţ̧at. \\ "Pour celui qui meurt dans n'importe quel voya- \\ ge." \\ non-A [2] Ithotcen eztirenenţ̧at. \\ "Pour ceux qui ne se noient pas." \\ [3] Lur bedincatu gabean ehorztean. \\ "Quand on enterre [un corps] en terre non bénie." \\ $<$ \\ [4] Vntcian hillic urera etchatcean. \\ "Quand on jette à l'eau un [homme] mort à bord \\ du navire." \\ [5] Itsassoan ithoentçat. \\ "Pour ceux qui se sont noyés en mer." \\ A [6] Vr bazterrean Aurkhituenţ̧at. \\ "Pour ceux qui ont été trouvés au bord de l'eau." \\ non-A [7]Aurkhitcen eztirenentçat. \\ "Pour ceux que l'on ne trouve pas." \\ [8] Eliçan ehorzten direnentçat. \\ "Pour ceux que l'on enterre dans l'église." \\ Itsassoco othoitcen çarratcea. \\ "Fermeture [du cycle des] prières pour la mer."
}

\title{
VI.3 - Tableau II
}

$A-B V R V I$
(1) A1
(2) $\mathrm{A2}$
(3) $\mathbf{A 3}$

$B-B V R V I I$

(4) B1

(5) $\mathrm{B} 2$

(6) B3

(7) $\mathrm{B} 4[(8) \mathrm{B} 4 \mathrm{a}-(9) \mathrm{B} 4 \mathrm{~b}-(10) \mathrm{B} 4 \mathrm{c}-(11) \mathrm{B} 4 \mathrm{~d}-(12) \mathrm{B} 4 \mathrm{e})$

(13) $\mathrm{B} 5$ [(14)B5a - (15)B5b]

(16) B6 


$\begin{array}{rr}C-B V R V I I I & \\ (17) & \mathrm{C} 1 \\ (18) & \mathrm{C} 2 \\ (19) & \mathrm{C} 3 \\ (20) & \mathrm{C} 4 \\ (21) & \mathrm{C} 5 \\ (22) & \mathrm{C} 6\end{array}$

D - BVRV IV

(23) D1

(24) D2

(25) D3

(26) D4

(27) D5

(28) D6

(29) D7

(30) D8

(31) D9

(32) $\quad \mathrm{D} 10$

(33) D11

(34) D12

(35) D13

(36) D14

(37) D16

(39) $\quad$ D17

(40) D18

$E-B V R V V$

1...] ABISVA

"AVIS"

(41) E1

(42) E2

(43) E3

(44) E4

(45) E5

(46) E6

(47) E7

(48) E8

(49) E9

F BVRV VI

(50) F1

(51) F2 


$\begin{array}{cc}G-B V R V V I J & \\ (52) & \text { G1 } \\ (53) & \text { G2 } \\ (54) & \text { G3 } \\ (55) & \text { G4 } \\ (56) & \text { G5 } \\ (57) & \text { G6 } \\ (58) & \text { G7 } \\ (59) & \text { G8 } \\ (60) & \text { G9 } \\ (61) & \text { G10 } \\ (62) & \text { G11 } \\ (63) & \text { G12 } \\ (64) & \text { G13 } \\ (65) & \text { G14 } \\ (66) & \text { G15 } \\ (67) & \text { G16 } \\ (68) & \text { G17 } \\ (69) & \text { G18 } \\ (70) & \text { G19 } \\ (71) & \text { G20 } \\ (72) & \text { G21 } \\ (73) & \text { G22 } \\ (74) & \text { G23 }\end{array}$

$\begin{array}{cc}H-B V R V V I I I & \\ (75) & \text { H1 } \\ (76) & \text { H2 } \\ (77) & \text { H3 }\end{array}$

I - BVRV IX

(78) I1

(79) I2 [(80) I 79a - (81) I79b]

$J-B V R V X$

(82) J1

(83) J2

(84) J3

(85) J4

(86) J5

(87) J6

(88) $\quad$ J7

(89) J8

[...] J9 


\section{Bibliographie}

AQUIEN Michèle (1993) : Dictionnaire de poétique, Librairie Générale Française.

BARKHAM Selma (1987) : "Los vascos en las pesquerias transtlánticas (1517-1713), in Itsasoa 3. Los vascos en el marco Atlántico Norie. Siglos XVI y XVII, éditions Etor.

BARTHES Roland (1971/1980) : Sade, Fourier, Loyola. Points Seuils, Paris 1980.

ETCHEBERRI (de Ciboure) Joannes (1627/1669/1978) : Manval devotionezcoa edo ezperen oren oro escvetan erabilltşeco liburutchoa... [l'ouvrage est divisé en deux parties], G. Millanges, Bordeaux, 1627 ; édition facsimilée de la seconde édition de 1669 chez I. Mongiron. Millanges à Bordeaux par les édi. tions Hordago, Donostia-San Sebastián en 1978 ; édition critique du premier livre par Patxi ALTUNA. Euskaltzaindia-Académie de la Langue Basque, Bilbao, 1981.

FRY Northrop (1981/1984): Le Grand Code. La Bible et la littérature. Traduction française avec préface de Tzvetan Todorov, Seuil, 1984

FUMAROLI Marc (1994) : L'âge de l'éloquence. Rhétorique et "res" literaria de la Renaissance au seuil de l'époque classique, Albin Michel.

HARITSCHELHAR Jean (1969) : Le poète souletin Pierre Topet-Etchahun (1786-1862). Contribution à l'étude de la poésie populaire basque du XIX $X^{\circ}$ siècle. Société des Amis du Musée Basque, Bayonne.

JAUPART Fernand. (1981) : Histoire Maritime de Saint-Jean-de-Luz et de Ciboure aux XVIF et au XVIIT siècles, tome I, XVII" siècle, tome II XVIII siècle. Chambre de Commerce et d'Industrie de Bayonne.

LAFITTE Pierre (1941): Le basque et la littérature d'expression basque en Labourd, Basse-Navarre et Soule, Librairie "Le livre", Bayonne.

LASSUS Alfred (1999) : "Trois lettres au sujet de la généalogie de Pierre Detcheverry Dorre", (inédit).

MATERRE Estève (1617/1693): Dotrina Christiana in Bouqueta Lore Diuinoena bereciac eta Duronea apeçac T.P.S.V. Aita Materren liburuari emendatuac... Bayonan Piarres Dussarat Liburu eguilea bai= than. Aprobationerequin. Edition de 1693, Bayonne.

MICHELENA Luis (1960/1988) : Historia de la Literatura Vasca. Réédition de la première édition in Erein, Donostia-San Sebastián.

MITXELENA Koldo (1988) : Euskal idazlan guztiak IV. Literaturaren inguruan I. Euskal Editorecn Elkartea, klasikoak, Zarautz.

MOLINIÉ Georges (1992) : Dictionnaire de Rhétorique. Librairie générale Française, Paris.

MOREAU Roland (1992) : "Célébrités à Ciboure", in Ciboure [ouvrage collectif], éditions Ekaina, SaintJean-de-Luz.

OIHENART Arnaud (1665/1967/1997) : "L'art poétique basque". Réédition de l'article de Pierre LAFITTE publié en 1967 dans Gure Herria (la lettre de 1665 d'OIHENART était jusqu'alors inédite) in revue Oihenart, 1997. Une traduction en basque de l'Art Poétique Basque, avec une critique de la lecture de P. LAFITTE, a été publiée par Patri URKIZU in Iker 8, éditions Euskaltzaindia-Académie de la Langue Basque, Bilbao 1994.

ORPUSTAN Jean-Baptiste (1996) : Précis d'Histoire Littéraire Basque, 1450-1950. Cinq siècles de littérature en basque, éditions Izpegi, Baigorri.

OYHARÇABAL Bernard (1996) : "Hitz eratorriak Materreren Dotrina Christiana delakoan (1617)", Lapurdum $\mathrm{n}^{\circ}$ 1, Bayonne.

OYHARÇABAL Bernard (1999) : "Les prologues auctoriaux des ouvrages basques des XVI et XVII siècles", à paraître in Lapurdum 1999 [monographie sur Pedro de Axular], Bayonne.

POUVREAU Sylvain (1663 et non daté), Dictionnaire basque-français. Manuscrit inédit, rubrique celtique/basque $n^{\circ} 7$ (n. d.) et $n^{\circ} 8$ (15 octobre 1663), Bibliothèque Nationale de Paris.

IMBACH Ruedi et MÉLÉARD Marie-Hélène (1986) : Philosophes médiévaux. Anthologie de textes philosophiques (XIII-XIV' siècles). Université de Fribourg, Suisse, éditions 10/18, Paris. 
SUHAMY Henri (1986), La Poétique, Presses Universitaires de France, Paris.

TODOROV Tzvetan (1987) : La notion de Littérature, éditions du Seuil, Paris.

TURGEON Laurier (1982): Pêches Basques en Atlantique Nord (XVIK-XVIII siècles), Doctorat de 3* cycle, Université de Bordeaux IIII.

VILLASANTE Luis (1961): Historia de la Literatura Vasca, éditions Sendo, Bilbao,

VINSON Julien (1891-1898/1983) : Essai d'une Bibliographie de la Langue Basque. Reimpresion de la edición de 1891. Tomes I et II, éditions Caja de Ahorros Vizcaina, Bilbao 1983.

ZUMTHOR Paul (1983) : Introduction à la poésie orale. Collection Poétique, éditions du Seuil, Paris, 


\section{Notes}

1. Julien Vinson écrit ceci : “... avant 1617, Jean d'Etcheberri était de Ciboure, car il signe ciburutar, M. Haristoy dit que Jean d'Etcheberri était de Ciboure et qu'il fut jusqu'en 1638 curé de cette localité. Il avait trois frères à savoir le médecin dont il est parlé dans les préliminaires du Manual et un marin dit Dorre, traducteur de Hoyarsabal. La date de la publication d'Jtxasoco nabigacionea paraîtrait cependant incompatible avec cette hypothèse." (Vinson $[898,545$.

2. Archives Municipales de Saint-Jean-de-Luz: Doc. 1/ H H1-2-7 (année 1689). Doc. $2 /$ H H 1-2-16 (année 1690). Doc. 3/ H H 2-16 (année 1690). Doc. 4/ H H 1-2-17 (année 1690).

Les quatre documents cités donnent la "Liste du nombre de mariniers quil ya dans la Communauté de St. Jean de Luz" [titre du doc. H H 1-2-16], et disent que Pierre Detcheverry dit Dorré, sieur de Chucutunia, est pilote et a 53 ans en 1689 (le mois n'est pas précisé) et en janvier 1690.

3. M. Alfred Lassus m'a aimablement communiqué les informations sur la généalogie de la famille de Piarres Detcheverry dit Dorre au XVII ${ }^{e}$ siècle (correspondance privée).

4. Certains critiques semblent penser que le dizain en latin de Stephanus Hirigoyti qui figure en tête du Manual... :

.... Equora qui sulcant dehent tibi plurima, naues

Quad tua fecit eis ingeniosa manus,

Quas furor ingentis Neptuni savius, et quid

In gremiis foueant terra, fretumque simul,

Non hominum liuor mordax abolebit : at ipsa

Etcheberri cum nomine semper erunt. (Altuna 1981, 37, cf, aussi la traduction en basque)

indique que Joannes Etcheberri a pu avoir une quelconque activité dans la fabrication des navires... Je pense pour penser que cette hypothèse est fort improbable. 11 me semble, en effet, que Stephanus Hirigoyti s'exprime d'une façon métaphorique. C'est pourquoi d'ailleurs les navires dont parle le Hirigoyti ne pourront jamais itru attints par l'ire de Neptune, et pourquot ils auront la même destinćc que le nom de Detcheberri qui vivra à jamais.

5. ... batek uste izan dezake orduko euskaldun alfabetatuetan ere arras gutik baizik ez zekiela euskaraz irakurtzen eta idazten. Ez da segur, hatatik, gauzak arras horrela konprenitu behar direla. Ezen euskara orduan letra hizkuntza guti arautua bazen ere, hura zatekeen jendeen arteko mintzabide nagusia Lapurdin, idatzian ere baliatua. Argitara eman dira berrikitan Urtubiako andereak 1598 an euskaraz idatzi gutun batzuk, Lapurdiko handikiek, behar zutelarik bederen, edo komeni zitzaienean, euskaraz izkiriatzen zutela erakusten digutenak (Floristan Imizkoz, 1993). Modu berean, 1620an edo hor nonbait argitara eman zituen Voltoirek euskararen ikasteko bere solasaldiak, Donibane Lohizunen euskara merkataritzako mintzaira gisa ere erabilia zela erakusten dutenak. Segurenaz ere, ordu arte euskarazko liburu guti publikatua izan bazen ere, aspaldi zuen euskara idatzia baliatua zela Lapurdiko gizartean, eta pentsa daiteke euskara zela idazterakoan ere lapurtar anitzi egiazki balia zekieken mintzaira bakarra.

"... on pourrait penser que même parmi le peu de Basques alphabétisés du temps, seule une toute petite minorité savait lire et écrire en basque. Il n'est cependant pas dit que les choses doivent être comprises de cette manière. Car, même si la langué basque était peu réglementée en tant́ que langue des lettres, il n'en était pas moins vrai qu'elle était l'outil de communication principal utilisé par les gens du Labourd, y compris comme langue écrite. Récemment, quelques lettres rédigées en basque en 1598 par la Dame d'Urtubie on été publiées : elles nous montrent que lorsqu'ils en éprouvaient le besoin ou le désir, les personnes importantes du Labourd écrivaient en basque (Floristan Imizkoz 1993). De même, ce fut autour de 1620, que Voltoire publia des dialogues pour apprendre le basque, dialogues qui révélaient qu'à Saint-Jean-deLuz, le basque était utilisé aussi comme langue commerciale. Même si peu de livres en basque avaient été publiés jusque là, cela faisait probablement longtemps que le basque écrit était utilisé dans la société labourdine, et nous sommes en droit de penser que la langue basque était l'unique langue qui pouvait réellement servir à nombre de Labourdins à l'heure d'écrire." (Oyharçabal 1996, 39).

6. Cf. mon article à paraitre in Lapurdum (monographie consacrée à Axular et à son époque) : Le routier des mariniers du Capitaine Martin de Hoyarsabal de Ciboure (1579), questionnement autour d'une énigme.

7. Voici ce que Julien Vinson dit du franciscain Esteve Materre :

"Il a publié en français, à Paris, en 1606, chez Sevestre, l'ouvrage suivant, cité, du reste, par Wadding : "I'horloge spiriuelle...[...]. Ce serait donc de 1606 à 1616 que le Père Materre aurait visité le pays basque et y aurait appris la langue du pays; il l'a étudiée à Sare, sans doute sous la direction d'Axular. [...]". 
Ailleurs il dit qu'il était "gardien du couvent des Franciscains ou Cordeliers de La Réole", en 1617 et qu'en 1623, "il était passé au couvent de Toulouse". (Vinson 1891, 54)

Au sujet du plus ancien exemplaire conservé de la Dotrina Christiana, celle de 1623, (1'édition se trouve à la bibliothèque Bodleyenne à Oxford) il ajoute

Notons que cette édition comporte une "approbation en basque" signée P. de Axular et datée de Sare, $1^{\circ}$ décembre 1616 Vale.

"Láp. 176 n'a que six lignes imprimées et tout le reste est blanc. Un ancien propriétaire du livre a écrit son nom dans cet espace blanc de la manière suivante : "Francis de Esparbem guataluna de Murguy. I617". Cette date de 1617 est singulière, puisque le livre est de 1623. Serait-ce 1637 ? ou 1757 ? ou avons-nous à faire à un exemplaire formé de deux autres dont l'un serait de la première édition? Si la date de 1617, pour cet ex-libris, est exacte et authentique, elle peut aussi s'expliquer d'une autre façon : elle prouverait en effet que l'édition de 1623 n'est pas à proprement parler une nouvelle édition, une réimpression de celle de 1617 , mais que pour la faire on aurait repris les exemplaires restants de la première dont on aurait refait le titre et auxquels on aurait ajouté les p. 177 et suivantes." (Vinson 1891, 53-54)

A propos du fragment dont je parle dans cet article Julien Vinson ajoute les précisions suivantes :

"Lespèce de "Directoire des Marins" dont parle Larramendi, se compose sans doute des deux morceaux : Marinelec nola egun oroz eguin behar dituzten bere Othoiţ̧ac itsasoan dabiltţan denboran, hala groicean nola arratsean eta Itsasoan tormenta ailltchatcen denean erran behar diren Oracinoac. "Comment les Marins doivent faire tous les jours leurs prières dans le temps où ils marchent sur la mer, le matin comrine le soiñ" et "Oraisons qui doivent être dites quand la tourmente s'élève sur la mer." (Vinson $1898,54)$

Toutes ces prières, dit Vinson, sont en prose et en basque, sauf celles des p. 210-211 et 354-384 qui sont en latin.

Comme on l'aura compris, l'édition de 1617 ne nous est pas parvenue, seules sont conservées l'édition de 1623 et celle que Villasante dit être de I 693 (Villasante 1961, 69). II s agit d'une édition avec des adjonctions ultérieures faite par "le prêtre Duronea" et qui s'intitule, BOVQLETA LORE DIVINOENA BERECIAC ETA DURONEA APECSAC T.PS. F. AITA MATERREN LIBURUARI emendatuac, imprimé à Bayonne par Pierre Dussarrat (en 1693 ?).

8. “... l'ouvrage de Materre [...] est peu connu car fort rare, du fait notamment de l'absence de réédition depuis bientôt trois siècles. Il s'agit pourtant, d'une part, du premier livrc basque écrit en prose qui ne soit pas une traduction (même s'il faut tenir compte de la nature particulière de l'ouvrage : un livre d'instruction religieuse associé à un recueil de prières), d'autre part, de la prennière cuuvre écrite en labourdin classique du XVII siècle.

La chose est d'autant plus remarquable que l'auteur, un franciscain venu en Labourd après 1606 , avait appris le basque à l'âge adulte et apparemment en l'espace de quelques années (il n'était plus en territoire basque en 1617) : c'est donc aussi le premier ouvrage écrit par un non-natif, et, pour dire vrai, s'il n'en avait pas fait la confidence dans un avertissement adressẻ aux Basques, c'est un fait que ia seule lecture de l'ouvrage n'aurait probablement pas permis de déceler. La langue, en effet, est claire, naturelle, à la fois simple et de très bonne tenue, en tous points préfiguratrice de cette école labourdine dont l'œuvre d'Axular marque le sommet." (Oyharçabal 1996, 71)

9. Danıs lc texte de l'édition de 1693 (Bouqueta... celle ayant appartenu à Julio de Lirquijo) que j'ai sous les yeux, sous la rubrique ITSASSOAN DABILCAN guztienţ̧at, "Pour tous ceux qui naviguent" avec mention de l'année 1693, certaines prières sont manuscrites (pages 455 à 458).

10. Dans l'édition de 1693 (Bouqueta...) que j'ai utilisée, il y avait inversion du caractère d'imprimerie " $u$ " pour " $n$ ",

1 I... Materrek bere liburuko ondar partean, ez du dudatzen itsas untzietan euskaraz irakurtzeko gai ziren mariñelak izanen zirela, eta haiek haren otoitz liburua eskuetan izanen zutela."

Dans la dernière partie de son livre, Materre ne doute pas que les marins embarqués sur les navires puissent lire, ni qu'ils auront son livre de prières entre les mains." (Oyharçabal 1996, 39)

12. cf. B. Oyharçabal 1999.

13. Traduction de B. Oyharçabal.

14. Traduction de B. Oyharçabal.

15. Traduction de B. Oyharçabal.

16. Traduction de B. Oyharçabal. 
17. Je souligne,

18. Dans la traduction le terme est au pluriel, mais Etcheberri utilise le terme générique de "couplet" cannlk, au singulier.

19. Je souligne.

20. Traduction de B. Oyharçabal.

21. "... si l'on opte pour un point de vue structural, chaque type de discours qualifie habituellement de littéraire a des "parents" non littéraires qui lui sont plus proches que tout autre type de discours "littéraire". Par exemple, une certaine poésie lyrique et la prière obéissent à plus de règles communes que cette même poésie et le roman historique Guerre et paix. Ainsi l'opposition entre littérature et non-littérature cide la place à une typologie des discours." (Todorov 1987, 25)

22. Pour Jean-Baptiste Orpustan, qui évoque aussi le contexte dans lequel ont pu évoluer Esteve Materre et Joannes Etcheberri, ce dernier fait partie des "poètes religieux":

"Dans ce même début du XVII" siècle des textes poétiques manuscrits de moindre importance apparaissent ici où là, religieux le plus souvent, parfois profanes, satiriques ou amoureux. Mais c'est à ce qu'on peut bien nommer "l'école littéraire basque luzienne", parce que groupée autour du port labourdin, alors en main d'armateurs dynamiques, et du couvent des Récollets que l'évêque Bertrand d'Etchauz venait de fonder à Ciboure en 1609, et dans son prolongement intérieur vers Sare où depuis 1600 officie Axular, que l'on doit un développement exceptionnel de la littérature religieuse en langue basque, dans la première moitié et le milieu du XVII ${ }^{c}$ siècle. Si la figure centrale de ce mouvement littéraire exclusivement clérical par l'appartenance des auteurs, est le même Axular, le maittre de la prose basque classique, il comprenait des poètes. Oyènart en 1665 cite "Monsieur d'Etcheberri prestre de Siboro" [...]" (apud Orpustan 1996, 42)

23. La traduction est de Julien Vinson.

24. Cf. LIBVRVAREN ERREPARTIMI IT AZ dans la traduction de B. Oyharçabal (1999):

De la composition du livre

"Ce volume tout enticr se compose de deux livres/ e premici enseigne les choses qu'il faut savoir/Le second les prières pour faire les dévotions/Que chaque cbrétien devrait dire.De même, le premier livre, en deux parties,/Ainsi que tu le verras, se divise/La premic̀re parle des choses communes/Que chacun doit savoir sur le bout des doigts,/Lautre ensuite des sept fins dernières de l'homme/Que le chrétien jour et nuit doit à la fin considérer/Le livre suivant de même conte deux choses,/Et enseigne les oraisons simples et doubles./Finalement le manuel est résumé/De manière à ce que quiconque puisse le lire avec peu de peine [...]"

25. Ce "Bourgeois" est bien entendu l'Armateur (celui qui "arme "le navire, c'est à dire qui se charge de l'équiper, "et de le faire sortir du port"), le terme burigesa peut d'ailleurs au XVIII siècle, par exemple, prendre ce sens, mais les deux versions du Dictionnaire basque-français de Pouvreau qui datent des années 1663 (I'une des versions porte cette date), 1665/1666? (pour la deuxième), indique : "Burgezza : bourgeois." (Celtique/Basque $\mathrm{n}^{\circ} 7$, archives de la Bibliothèque Nationale de Paris - Richelieu-).

26 Littéraliment. "des chefs".

27. "Le Maître a pouvoir sur tout le personnel, règne sur la coque et sur le gréement, veille au lestage. Il commande l'appareillage et le mouillage ainsi que la manœuvre des ancres." (Jaupart 1981, tome II).

28. Le Colonel Fernand Jaupart fournit les précisions suivantes:

"Le Pilote joue un rôle essentiel. Le Pilote Principal, sur les gros navires, est le "navigateur" qui établit et suit la route du navire, dans la direction et pour la destination prévues.

Il note sur la carte le chemin parcouru ; il dispose de plusieurs appareils : la boussole, le loch pour se rendre compte de la vitesse, des sabliers faisant fonction d'horloge.

Il conseille en ce qui concerne le déploiement de la voilure, soit pour l'augmenter, soit pour le diminuer, selon la force ou la direction des vents."

"Le Capitaine [...] s'en remet au pilote [en ce qui concerne la navigation]. En cas de danger ou de situation critique, il prend l'avis du Pilote et des "Anciens"."

"Il faut se rendre compte de l'importance de son rôle, surtout à l'approche des côtes et dans la navigation de nuit, à une époque où les phares étaient rares et leur portée lumineuse faible (ils étaient en général chauffés au bois).

Pour être admis à cette fonction et titre, il [le Pilote] doit subir un examen et justifier d'un temps minimum de navigation." (Jaupart 1981, tome II)

Le Vice-Amiral Willaumez dans son Dictionnaire de Marine indique : 
"On en distingue de trois espèces : celui qui exerce le pilotage en pleine mer est le plus instruit ; c'est le pilote hauturier naviguant sur toutes les mers. Le titre et le grade de pilote hauturier sur les bâtimens de guerre ont été supprimés en 1791 - Le pilote côtier est ordinairement un maître ou patron, reçu pour le petit cabotage. Le pilote lamaneur ne prend les navires qu'aux entrées des rivières ou des ports." (Willaumez, 1831, 445)

29. La route "est la ligne, sur le compas de route, que suit un bâtiment à la mer, ou la direction de l'air de vent qui doit le conduire d'un lieu à un autre, si le vent ne l'empêche pas de porter en droite route, de mettre en route, de gouverner la route. On donne la route au timonier, en lui désignant l'air de vent sur le compas où il faut qu'il gouverne." (Willaumez 1831, 508).

30. "Prendre hauteur à la mer, c'est mesurer avec un instrument astronomique, l'élévation du soleil au dessus de l'horizon, lorsqu'il passe au méridien, pour avoir la latitude ou la hauteur du pôle. On prend des hauteurs absolues dans les intervalles du lever de l'astre, à midi et quelques heures avant son coucher; il faut préférer l'instant du passage du soleil au premier vertical. - On prend des hauteurs correspondantes; ce sont celles prises à distances égales du méridien. - On dit qu'on est à la hauteur d'un cap, d'une île, lorsqu'on est près sur son parallèle. (Willaumez $183 \mathrm{I}, 333$ )

31. Pour Lemacoa, Sylvain Pouvreau donne la signification suivante dans son dictionnaire manuscrit du XVII siècle: "nauclerus, celuy qui tient le gouvernail. lemaria [...]". Celtique/Basque n 7 , archives de la Bibliothèque Nationale de Paris (Richelieu). J'utiliserai pour ma part, la périphrase de Pouvreau, plutôt que le seul "timonier."

32. "Autrefois les timoniers étaient une classe d'hommes désignés sur les rôles d'équipages des bâtimens du roi pour le service de la timonerie, ils dépendaient du maître pilote qui surveillait la route donnée, et faisait gouverner par ces hommes expérimentés, particulièrement propres au service de la roue de gouvernail. Un bon timonier est un homme précieux dans le mauxais toms, les chasses etc., et les mcilleurs matelots d'un bâtiment doivent être les meilleurs timoniers." (Willaume\% 1831, 546).

33. Il est clair que lema ne doit pas être entendu ici dans le sens de "timon" ou "gouvernail". L'utilisation de l'inessif -n (leman) indique qu'il s'agit bien d'un lieu, d'un habitacle où se trouve la barre de gouvernail te où se place le timonier. Je le traduirai done par "timonerie", mais ni Lhande, ni Azkue ne montionnent coilus signitïcation.

Au XVIII" siècle, la "timonerie" se situe sur le pont; il en était probablement de même au XVII" sićcle puisque le Colonel Jaupart précisi que :

"Quelle que soit la catiçorie à laquelle il appartient, le navire du XVIII" siècle - tout comme colui du XVII - a sensiblement la meme forme générale : vu de face, il précnte des flancs renflés ; latérulement, il est d'une hauteur progressivement augmentée, de l'avant vers l'arrière, où se dressent plusieurs étages superposés (on en compte jusqu'à cinq sur les gros bâtiments)."

La timoneric se trouve done sur le pont, à l'étage situce sous la "dunette" "petite construction surélevée" où sont les chambres des officiers et ćcntucllement celles des gens importants); la plupart du temps, la salle dénommée "corps de garde" sert de "timoneric"

"Le "corps de garde" termine ver. l'avant cette construction. [...]. Ce corps de garde sert aussi à loger des canons. même dans les bâtimenis marchands.

Il est surtout cmployé à usage de timonerie. C'est là que se tient l'homme de barre, qui manœuvre celle-ci au moyen d'un grand levicr vertical, actionnant la barre hori/ontale [...]. Dans cette timonerie existe un ensemble d apparcils utilisćs pour la navigation." (Jaupart 1981, tome I1)

34. "Etchera bihurtica. Retourner a la maison." (Sylvain Pouvreau, in op. cit. $n^{\circ} 7$ ).

35. "Leihorra. terre ferme." (Sylvain Pouvreau, in op. cit. n 7 et $n^{\circ} 8$ ).

36. "Cembait. quelqu'un." "Cembaiter cran du. quelqu' un a dit." (Sylvain Pouvrea, in op. cit. $n^{\circ} 8$ ).

37. La position de la ponctuation peut induire en erreur. L A titre général du chapitre qui annonce la thématique des nouvelles prières, devrait être ponctué après othoitçac. Le premier complément circonstanciel de temps egunaz (du jour) est à relier au sccond gauaz (de la nuit), une prière étant consacrée à chaque unité temporelle.

38. Il s'agit, bien entendu, des Saint (c)s l'atron(ne)s de la mer. 